\title{
Naringenin Regulates FKBP4/NR3C1/TMEM173 Signaling Pathway in Autophagy and Proliferation of Breast Cancer and Tumor-Infiltrating Dendritic Cell Maturation
}

Hanchu Xiong ( $D$ 11718266@zju.edu.cn )

Zhejiang Provincial People's Hospital https://orcid.org/0000-0001-6075-6895

\section{Zihan Chen}

First Hospital of Zhejiang Province: Zhejiang University School of Medicine First Affiliated Hospital

Baihua Lin

Zhejiang Provincial People's Hospital

\section{Cong Chen}

Zhejiang University School of Medicine Sir Run Run Shaw Hospital

\section{Zhaoqing Li}

Zhejiang University School of Medicine Sir Run Run Shaw Hospital

\section{Yongshi Jia}

Zhejiang Provincial People's Hospital

\section{Linbo Wang}

Zhejiang University School of Medicine Sir Run Run Shaw Hospital

Jichun Zhou

Zhejiang University School of Medicine Sir Run Run Shaw Hospital

\section{Research}

Keywords: FKBP4, TMEM173, Autophagy, Exosome, Dendritic cell, Breast cancer

Posted Date: July 7th, 2021

DOI: https://doi.org/10.21203/rs.3.rs-659646/v1

License: (9) (1) This work is licensed under a Creative Commons Attribution 4.0 International License.

Read Full License 


\section{Abstract \\ Background}

TMEM173 is a pattern recognition receptor detecting cytoplasmic nucleic acids and transmits cGAS related signals that activate host innate immune responses. It has also been found to be involved in tumor immunity and tumorigenesis.

\section{Methods}

Bc-GenExMiner, PROMO and STRING database were used for analyzing clinical features and interplays of FKBP4, TMEM173 and NR3C1. Transient transfection, western blotting, quantitative real-time PCR, luciferase reporter assay, immunofluorescence and nuclear and cytoplasmic fractionation were used for regulation of FKBP4, TMEM173 and NR3C1. Both knockdown and overexpression of FKBP4, TMEM173 and NR3C1 were used to analyze effects on autophagy and proliferation of breast cancer (BC) cells. Flow cytometry analysis, cytokine analysis and exosome isolation and identification were utilized to test tumorinfiltrating dendritic cell (TIDC) maturation.

\section{Results}

In this study, we firstly identified that FKBP4/NR3C1 axis was a novel negative regulator of TMEM173 in BC cells. The effect of FKBP4 appeared to be at the transcriptional level of TMEM173 since it could suppress the promoter activity of TMEM173, thereby affecting TMEM173 at mRNA and protein levels. Bioinformatics and in vitro experiments further demonstrated that FKBP4 regulated TMEM173 via regulating nuclear translocation of $\mathrm{NR3C} 1$. We then reported that naringenin, a flavonoid, could enhance autophagy and suppress proliferation of BC cells through the induction of TMEM173 in vitro and in vivo. Naringenin was also found to promote TIDC maturation through FKBP4/NR3C1/TMEM173 axis of both $\mathrm{BC}$ cells exosome and DC itself.

\section{Conclusion}

We demonstrated that naringenin could induce cell proliferation inhibition and cytoprotective autophagy of $B C$ cells and enhance TIDC maturation, at least in part, though regulation of FKBP4/NR3C1/TMEM173 signaling pathway. Identification of FKBP4/NR3C1 axis as a novel TMEM173 regulator would provide insights for novel anti-tumor strategy against $\mathrm{BC}$ among tumor microenvironment.

\section{Background}

Breast cancer (BC) is a leading cause of cancer-related deaths in women aged 40 years and younger[1]. Early detection and comprehensive treatments, which consist of surgery, radiation, chemotherapy, 
endocrine therapy and targeted therapy, have dramatically improved the prognosis of BC patients. In recent years, immunotherapy in BC showed promising future. Cancer vaccines, bispecific antibodies, and immune checkpoint inhibitors are verified to have potential applied value in BC immunotherapy[2]. For instance, adaptive immune checkpoint therapies by targeting cytotoxic T-lymphocyte antigen-4, programmed cell death-1 (PD-1) and ligand partner for PD-1 (PD-L1) for BC have been used in clinical trial[3, 4]. Nevertheless, a portion of $B C$ patients still cannot benefit from above-mentioned immunotherapy strategies[5]. Therefore, unravelling the potential molecular mechanisms of both innate and adaptive immune system in $\mathrm{BC}$ cells is essential to further understand and improve immune related anti-tumor effects.

Transmembrane protein 173 (TMEM173), also named stimulator of interferon genes (STING), residing in the endoplasmic reticulum (ER), has early been identified as a critical adaptor for cyclic dinucleotides (CDNs) produced from a cellular nucleotidy Itransferase referred to as cyclic GMP-AMP synthase (cGAS) and regulates the induction of numerous host defence gene[6-8]. Therefore, TMEM173 has been found involved in anti-microbial innate immunity, as well as in the pathogenesis of some autoimmune disorders $[9,10]$. Recently, several studies revealed the suppressive function of TMEM173 in tumorigenesis, including BC, gastric cancer, leukemia, prostate cancer, colorectal cancer, melanomas and so forth[11-16]. Our previous team's study has discovered that HIV-2/SIV Vpx acts as a novel inhibitor of innate immune activation associated with TMEM173 signalosomes[17], we are wondering whether and how TMEM173 performs its anti-tumor effects by connection with novel molecular chaperones in BC.

As one of the most extensively studied proteins among the 18 identified human FK506-binding proteins (FKBPs), FK506-binding protein 4 (FKBP4), also known as FKBP52, has been reported to exhibit multiple functions which involve binding to different cellular receptors or targets in various kinds of cancers[1822]. For example, FKBP4 has been demonstrated to interact with heat shock protein 90 (Hsp90) to affect steroid hormone receptor function in $\mathrm{BC}[23]$. In terms of immune regulation, phytanoyl-CoA alphahydroxylase (PAHX) has been regarded as a specific target of FKBP4 for studying the cellular signaling pathway in the presence of immunosuppressant drugs[24]. Our previous work found that FKBP4 interacted with non-coding RNAs and mRNAs during the occurrence and development of $\mathrm{BC}$, thus playing a role in promoting cancer[22, 25]. Nevertheless, current immunologic mechanism of FKBP4 is still in its infancy for $\mathrm{BC}$, it is necessary to explore more new detailed contents of its regulation of innate and adaptive immunity functions during the occurrence and development of $\mathrm{BC}$.

The NR3C1 (nuclear receptor subfamily 3, group C, member 1/glucocorticoid receptor) normally resides in the cell cytoplasm, the NR3C1 protein translocates to the nucleus when bound to glucocorticoids, thus involved in growth, reproduction, metabolism, immune and inflammatory reactions, as well as central nervous system and cardiovascular functions and tumor cellular proliferation and differentiation[26]. Researches on NR3C1 and BC have also been conducted in recent years, e.g., high levels of NR3C1 expression and high concentrations of cortisol have been shown to have an anti-proliferative effect in cancerous breast tissue[27]. Some studies have preliminarily found that NR3C1 is associated with FKBPs, but the specific mechanisms remain unclear in $\mathrm{BC}[28]$. 
Exosomes, also termed small extracellular vesicles, have a diameter ranging from 40 to $150 \mathrm{~nm}$, which are secreted by different types of cells and contain various cargoes, including DNA, mRNA, noncoding RNA, proteins and so forth[29]. Currently, researches on the role of exosomes involved in cancer progression have grown exponentially, including immune regulation, which suggest that cancer cells could secrete large numbers of exosomes to regulate innate and adaptive immune cells among tumor microenvironment for immune escape[30]. For instance, a study by Möller et al. showed that highly metastatic murine BC cells derived exosomes directly suppressed T-cell proliferation and inhibited NK cell cytotoxicity[31]. Although Demaria et al. identified exosome as a mechanism whereby DNA was transferred from irradiated BC cells to tumor-infiltrating dendritic cell (TIDC)[32], whether and how FKBP4 or TMEM173 participated in exosomes secreted by $\mathrm{BC}$ cells might regulate $\mathrm{DC}$ maturation and function remain to be explored.

In this study, we showed that naringenin, a flavonoid shown anti-tumor effects in various carcinomas in other studies[33], promoted autophagy and suppressed proliferation via new-found FKBP4/NR3C1/TMEM173 signaling pathway in luminal A and basal-like subtype of BC cells. Meanwhile, naringenin also could trigger $\mathrm{BC}$ cells to excrete exosomes to TIDC among tumor microenvironment through regulating FKBP4/NR3C1/TMEM173 signaling pathway, thus leading to maturation and activation of TIDC. Mechanically, these effects relied on downregulation of FKBP4, which transcriptionally upregulated TMEM173 through intensive nuclear translocation of NR3C1. Identification of FKBP4/NR3C1 axis as the novel TMEM173 transcriptional regulator would provide in-depth insights for immunological anti-tumor strategy to overcome BC.

\section{Materials And Methods 2.1 Cell culture}

MCF10A, MCF7, T47D, BT549, BT474, SKBR3 cells were obtained from the American Type Culture Collection (ATCC). MCF10A was cultured in Mammary Epithelial Basal medium. MCF7 was cultured in Eagle's Minimum Essential medium. T47D and SKBR3 were cultured in Dulbecco's modified Eagle's medium. BT549 and BT474 were cultured in Roswell Park Memorial Institute (RPMI) medium. Growth media were supplemented with $10 \%$ fetal calf serum and penicillin/streptomycin ( 100 units per $\mathrm{mL}$ ). All human cell lines were cultured at $37^{\circ} \mathrm{C}$ in a humidified incubator supplied with $5 \% \mathrm{CO}_{2}$.

\subsection{Antibodies and reagents}

Antibodies were used in the following dilutions: TMEM173 (1:1000, Proteintech, \#19851-1-AP), FKBP4 (1:1000, Proteintech, \#10655-1-AP), NR3C1 (1:1000, Proteintech, \#24050-1-AP), P62 (1:1000, MBL, \#PM045), Beclin1 (1:1000, CST, \#3495S), LC3B (1:1000, Sigma, \#L7543), Histone (1:1000, CST, \#3638), GAPDH (1:1000, Proteintech, \#60004-1-lg), Flag (1:1000, Sigma, \#F3165), HA (1:1000, Biolegend, \#901514). CD63 (1:1000, Abcam, \#ab134045), CD81(1:1000, Abcam, \#ab79559), TSG101(1:1000, Abcam, \#ab125011), Alix (1:1000, Abcam, \#ab88388). Secondary antibody goat anti-mouse (1:2500, HuaBio, \#HA1006), secondary antibody goat anti-rabbit (1:2500, HuaBio, \#HA1001). Anti-RABBIT IgG (H\&L) (GOAT) Antibody Rhodamine Conjugated (1:200, MULTISCIENCES, \#RK-611-1002), Anti-RABBIT IgG (H\&L) 
(GOAT) Antibody ATTO 488 Conjugated (1:200, MULTISCIENCES, \#RK-611-152-122S). Anti-Mouse CD11c, PE (1:20, MULTISCIENCES, \#AM011C04), Anti-Mouse CD86 (B7-2), APC (AM08605), Anti-Mouse CD80 (B71), FITC (AM08001), Anti-Mouse CD40, FITC (RK-200-302-N67). Naringenin was purchased from APExBIO (\#N1370), and resolved in DMSO at 10mM.

\subsection{Gene silence}

To validate hits from the genetic screens, breast cancer cells were transduced with pLKO.1 vectors, which in addition to the shRNA cassette carried a puromycin resistance cassette (pLK0.1-puro, Addgene plasmid \#10878). The shRNAs against TMEM173 and NR3C1 were cloned into pLK0.1 vectors using the Age1 and EcoR1 restriction sites. The shRNA targeting sequences of TMEM173[8] and NR3C1[34] were from published articles, lentiviral particles were produced as follows. In brief, HEK293T packaging cells were transfected with $800 \mathrm{ng}$ pLKO.1 DNA in combination with the packaging plasmids $200 \mathrm{ng}$ lenti-VSV-G, 400 ng lenti-RRE and $140 \mathrm{ng}$ lenti-REV. Virus containing supernatant was harvested at 36 and $48 \mathrm{~h}$ after transfection and filtered through a $0.45 \mu \mathrm{M}$ syringe filter with the addition of $10 \mu \mathrm{M}$ DEAE. Supernatants were used to infect target cells in another $12 \mathrm{~h}$ period.

\subsection{Western blotting and co-IP}

Knockdown efficiencies and biochemical responses were analyzed by western blotting. Cells were lysed in RIPA lysis buffer (EMD Millipore Corp.), supplemented with protease inhibitor and phosphatase inhibitor cocktail tablets (Roche). Separated proteins were transferred to nitrocellulose filter membranes and blocked in $5 \%$ milk in Tris-buffered saline, with $0.05 \%$ Tween-20. Immunodetection was done with various primary antibodies. Appropriate horseradish peroxidase-conjugated secondary antibodies were used and signals were visualized with enhanced chemiluminescence (Proteintech) by Tanon-5200 Chemiluminescent Imaging System (Tanon, China). Cells for co-IP were lysed in lysis buffer (50 mM Tris, $\mathrm{pH} 7.5$, with $150 \mathrm{mM} \mathrm{NaCl}, 0.5 \% \mathrm{NP}-40$, and protease inhibitor and phosphatase inhibitor cocktail tablets (Roche) at $4^{\circ} \mathrm{C}$ for $30 \mathrm{~min}$. After sonication and centrifugation, cell lysates were incubated with beads (Sigma) at $4^{\circ} \mathrm{C}$ overnight on a rotator. After six washes with wash buffer $(20 \mathrm{mM}$ Tris, pH 7.5, $100 \mathrm{mM}$ $\mathrm{NaCl}, 0.05 \%$ Tween-20, $0.1 \mathrm{mM}$ EDTA), $50 \mu \mathrm{L}$ of elution buffer (100 mM glycine-HCl, pH 2.5) was added to resuspend the beads, and the eluted proteins were obtained by centrifugation, followed by SDS-PAGE and immunoblotting analysis.

\subsection{Quantitative RT-qPCR}

Total RNA was extracted from cells by using TRIzol (Invitrogen). Reverse transcription was carried out with a 40- $\mu$ l volume by using a PrimeScript ${ }^{\text {TM }}$ RT Master Mix kit (TaKaRa) according to the manufacturer's instructions. Quantitative real-time PCR (qPCR) was carried out on an Applied Biosystems Fast 7500 machine by using a TB Green® Premix Ex Taq ${ }^{\text {TM }} \|$ kit (TaKaRa) and the following primer sets were used for qPCR analysis: TMEM173, 5'-GAGAGCCACCAGAGCACA-3' (forward) and 5'-TAGATGGACAGCAGCAACAG-3' (reverse), FKBP4, 5'- CATTGCCATAGCCACCATGAA-3' (forward) and 5'-TCCAGTGCAACCTCCACGATA-3' (reverse), NR3C1, 5'-AGTGGTTGAAAATCTCCTTAACTATTGCT-3' (forward) and 5'GGTATCTGATTGGTGATGATTTCAGCTA-3' (reverse), GAPDH, 5'-ATGACATCAAGAAGGTGGTG-3' (forward) 
and 5'-CATACCAGGAAATGAGCTTG-3' (reverse) as a control. The qPCR assay was carried out with a 15- $\mu$ l volume consisting of $7.5 \mu \mathrm{l}$ of a $2 \times$ TB green mix solution, $0.3 \mu \mathrm{l}$ of $10 \mu \mathrm{M}$ of each oligonucleotide primer, $0.3 \mu \mathrm{l}$ of ROX Reference Dye II and $2 \mu \mathrm{l}$ of the cDNA template. Target fragment amplification was carried out as follows: $95^{\circ} \mathrm{C}$ for $30 \mathrm{~s}$, followed by 40 cycles consisting of $95^{\circ} \mathrm{C}$ for $5 \mathrm{~s}$ and $60^{\circ} \mathrm{C}$ for $34 \mathrm{~s}$. Meltingcurve analysis was carried out at $90^{\circ} \mathrm{C}$ for $15 \mathrm{~s}$ and then at $60^{\circ} \mathrm{C}$ for $1 \mathrm{~min}$ and $95^{\circ} \mathrm{C}$ for $15 \mathrm{~s}$.

\subsection{Plasmids}

TMEM173-Flag, VR1012-HA, the control plasmid Renilla luciferase vector and pGL3-Basic luciferase vector were kindly provided by Xiao-Fang Yu (Zhejiang University, China), FKBP4 (GenBank accession number 2288) and NR3C1 (GenBank accession number 2908) were cloned into the VR1012-HA vector using the Sall and BamHI sites. The TMEM173 luciferase reporter plasmid was constructed by Shanghai Generay Biotech Co., Ltd.

\subsection{Luciferase reporter assay}

Breast cancer cells were plated into 12-well dishes and transfected the following day. $1 \mu \mathrm{g}$ of the reporter plasmid for TMEM173 promoter, 50 ng the Renilla luciferase control plasmid, and the indicated amounts of the expression plasmids were used per well. At $24 \mathrm{~h}$ posttransfection, luciferase activities were then measured by using a Dual-Luciferase reporter assay system (Promega) according to the manufacturer's instructions. Firefly luciferase activity was normalized to Renilla luciferase activity. Finally, the relative luciferase activities were expressed as fold changes over the empty-plasmid-transfected controls.

\subsection{Immunofluorescence}

T47D or BT549 cells grown on coverslips were fixed for 15 min with $4 \%$ paraformaldehyde in PBS, permeabilized for $10 \mathrm{~min}$ in $0.1 \%$ Triton X-100 in PBS, and blocked using 5\% BSA for $1 \mathrm{~h}$. The cells were then incubated with primary antibodies at $4^{\circ} \mathrm{C}$ overnight. After a rinse with PBS, the cells were incubated with fluorescent-conjugated secondary antibodies for $1 \mathrm{~h}$ at $37^{\circ} \mathrm{C}$. The nuclei were counterstained with 4 , 6-diamidino-2-phenylindole (DAPI; Sigma-Aldrich). Images were captured using a ZEISS laser scanning confocal microscope (LSM710; Zeiss). ZEISS ZEN Microscope software was used for acquisition.

\subsection{Autophagy flux monitoring}

To evaluate the formation of fluorescent LC3B puncta, p-mCherry-C1-EGFP-hLC3B (LC3B) was used to monitor autophagy flux, $48 \mathrm{~h}$ after LC3B co-transfection with siRNAs, the cells were washed with 1X PBS and immediately analyzed via confocal microscopy (magnification, $x 100$ ). The nuclei were counterstained with 4, 6-diamidino-2-phenylindole (DAPI; Sigma-Aldrich). Images were captured using a ZEISS laser scanning confocal microscope (LSM710; Zeiss). ZEISS ZEN Microscope software was used for acquisition.

\subsection{Nuclear and cytoplasmic fractionation}

T47D or BT549 cells were transfected with the indicated siRNAs for $72 \mathrm{~h}$, then cells were harvested, and the nuclear and cytoplasmic fractions were separated using Thermo Fisher Scientific NE-PER Nuclear and 
Cytoplasmic Extraction Reagents (78833) according to the manufacturer's protocol.

\subsection{Transient transfection}

Breast cancer cells cultured in 12-well tissue culture plates were transiently transfected with plasmids using Lipofectamine ${ }^{\circledR} 2000$ Reagent (Invitrogen) or siRNAs using Lipofectamine ${ }^{\circledR}$ RNAiMAX Reagent (Invitrogen) as instructed by the manufacturer. The siRNA targeting FKBP4, NR3C1 and TMEM173, as well as negative control siRNA were purchased from RIBBIO (Guangzhou, China). $72 \mathrm{~h}$ later, the whole-cell extract was prepared for RT-qPCR or western blot analysis.

\subsection{Cell proliferation assay}

Cell proliferation was analyzed using a Cell Counting Kit-8 (CCK-8) (DOJIND0). All cells were seeded into 96-well plates at a density of 5000 cells/well in a $100 \mu \mathrm{l}$ volume and incubated at $37^{\circ} \mathrm{C}$ under $5 \% \mathrm{CO}_{2}$ for $24,48,72 \mathrm{~h}$, followed by the addition of $10 \mu \mathrm{l}$ of CCK-8 solution. The absorbance in each well was measured after $1 \mathrm{~h}$ incubation using a microculture plate reader at a test wavelength of $450 \mathrm{~nm}$. Three replicate wells were set up in each group, and three independent experiments were performed.

\subsection{Colony formation assay}

Five hundred cells per well of breast cancer were seeded in a 6-well plate for colony formation assay. Two weeks after, they were fixed with $4 \%$ paraformaldehyde and stained with Crystal Violet. Colonies were quantified using ImageJ software.

\subsection{Generation of bone marrow cells}

The DC cells were generated from bone marrow stem cells of C57 mice, as previously described[35]. The cells were cultured in RPMI medium with $10 \%$ FBS, penicillin/streptomycin (100 units per $\mathrm{mL}$ ), GMCSF (20 $\mathrm{ng} / \mathrm{mL}$ ) (Signalway Antibody, \#AP73338), and IL4 (10 ng/mL) (Signalway Antibody, \#AP73338).

\subsection{Flow cytometry analysis}

Single cell suspensions were surface-labeled using the mAbs mentioned above for $30 \mathrm{~min}$ at $4^{\circ} \mathrm{C}$. After using Flow cytometry Staining buffer (MULTI SCIENCES, China), cells were analyzed with a BD FACSCalibur Flow Cytometer (Becton Dickinson). Cytometry data was analyzed using FlowJo software version 10.

\subsection{Cytokine Analysis}


Concentrations of IL-6, IL-12, TNF-a, IL-18, IL-1 $\beta$, and MCP-1 secreted by TIDC from each sample were determined by ELISA according to the manufacturer's instructions (eBioscience, San Diego, CA, USA). Cytokine concentrations are expressed as $\mathrm{pg} / \mathrm{ml}$.

\subsection{In vivo tumor xenograft assays}

Six-week-old male BALB/c nude mice were purchased from Vital River Laboratory Animal Technology Co, Ltd (Beijing, P.R. China). Animal experimental procedures were approved by the Medical Ethics Committee of Shandong University (ECAESDUSM 2014056). The six-week-old male mice were randomized into different groups. T47D shControl and T47D shTMEM173 cells $\left(5 \times 10^{6}\right.$ cells/mice) were implanted subcutaneously into the flank of nude mice. After tumor formation, naringenin $(50 \mathrm{mg} / \mathrm{kg}$ ) was administered orally to its respective treatment groups of animals for 28 days. Tumor volume $\left(\mathrm{mm}^{3}\right)$ was measured every three days and calculated by the formula (length $\times$ width $\times$ width) $/ 2$. When the tumors had reached a volume of approximately $600 \mathrm{~mm}^{3}$, the mice were euthanized, and the tumors were excised and embedded in paraffin for IHC analysis.

\subsection{Exosome isolation and identification}

BC cells were cultured in exosome-depleted $(160000 \mathrm{~g}, 16 \mathrm{~h})$ complete medium, and the supernatant was collected after $72 \mathrm{~h}$. After three successive centrifugations at $300 \mathrm{~g} \mathrm{(5} \mathrm{min),} 1200 \mathrm{~g}$ (20 min) and $10000 \mathrm{~g}$ (30 $\mathrm{min}$ ) at $4^{\circ} \mathrm{C}$, exosomes were purified with Amicon Ultra-15 centrifugal filters with Millex-GP syringe membrane filters with a $0.22 \mu \mathrm{m}$ pore size (Millipore Express) to eliminate debris and then centrifuged at $100000 \mathrm{~g}\left(70 \mathrm{~min}\right.$ at $\left.4^{\circ} \mathrm{C}\right)$. Vesicles resuspended were loaded onto the top of a step gradient comprising layers of $0.25,0.5,1.0,1.25$ and $2 \mathrm{M}$ sucrose (S9378, Sigma). The gradients were centrifuged at $100000 \mathrm{~g}$ for $2.5 \mathrm{~h}$ using a Beckman SW41Ti rotor. Ten fractions of $1 \mathrm{ml}$ were collected and separately washed with PBS at $100000 \mathrm{~g}$ for $2.5 \mathrm{~h}$ using a Beckman MLS50 rotor. The protein content of the exosomes was determined by using a BCA protein assay kit (Pierce, Pierce County, WA). The isolated exosomes were diluted in PBS and analyzed using a Nanoparticle Tracking Instrument (Particle Metrix, Germany) according to the manufacturer's protocol. The size and concentration of exosomes were analyzed using the Zetaview Analysis software.

\subsection{Bioinformatics analysis}

The expression, correlation and prognostic module of Breast Cancer Gene-Expression Miner v4.4 (bcGenExMiner v4.4) (bcgenex.centregauducheau.fr) were used to evaluate the expression, correlation and prognostic merit of FKBP4, NR3C1 and TMEM173 in human breast cancer. Transcription factors in TMEM173 promoter were predicted by PROMO (http://alggen.Isi.upc.es/cgibin/promo_v3/promo/promoinit.cgi?dirDB=TF_8.3). Protein to protein interacting network was analyzed by STRING (https://string-db.org/). 


\subsection{Statistics}

Two-tailed student's t-test was used in this study. Data shown was mean \pm SD from at least three independent experiments. Statistical probability was expressed as ${ }^{\star} p<0.05,{ }^{\star \star} p<0.01,{ }^{\star \star \star} p<0.001$.

\section{Results}

\subsection{Negative correlation of FKBP4 and TMEM173 in breast cancer}

Firstly, we used bc-GenExMiner v4.4[36], an online public tool focused on BC, to explore the clinicopathological characteristics of FKBP4 and TMEM173. Upregulated FKBP4 was found significantly related to luminal $A$, luminal $B$ and basal-like subtype of $B C$ patients than the normal group (Figure $1 A$ ), and downregulated TMEM173 was significantly related to luminal A, luminal B and basal-like subtype patients than the normal group (Figure 1B). Furthermore, we validated a significant negative association between FKBP4 and TMEM173 both in luminal $A$ and basal-like subtype of BC patients (Figure 1C, D), but not in luminal $B$ and HER2-positive subtype of BC patients (Figure S1). Then, the prognostic merits of FKBP4 and TMEM173 in luminal A and basal-like subtype of BC patients were further analyzed by using bc-GenExMiner v4.4, the Kaplan-Meier curve showed that increased levels of FKBP4 and decreased levels of TMEM173 were strongly correlated with worse overall survival both in luminal A and basal-like subtype of BC patients (Figure S2).

We also tested five BC cell lines (MCF7, T47D, BT549, BT474 and SKBR3) as well as normal breast cell line (MCF10A) for expression levels of FKBP4 and TMEM173. As shown in Figure 1E, expression of FKBP4 and TMEM173 were negatively correlated in BC cell lines, especially in T47D (representing luminal A subtype of BC) and BT549 (representing basal-like subtype of BC) cell lines. Since naringenin has been reported involved in immunity regulation[37], we wondered its effect of pharmacologically modulating TMEM173 expression. As shown in Figure 1F, naringenin significantly decreased FKBP4 expression and increased TMEM173 expression in T47D and BT549 cells.

\subsection{FKBP4 negatively regulates TMEM173 at protein, mRNA and transcription level}

To confirm the specific interaction between FKBP4 and TMEM173 at the molecular level, we firstly used siRNA specifically targeting FKBP4 in T47D and BT549 cells, which led to upregulation of TMEM173 at protein level (Figure 1G). In addition, we transfected FKBP4-HA plasmid in T47D and BT549 cells, which resulted in downregulation of endogenous TMEM173 (Figure 1H). These results clearly indicated that FKBP4 had a role in negatively regulating TMEM173 protein expression. We also found silencing of FKBP4 led to upregulation of TMEM173 at mRNA level in T47D and BT549 cells (Figure 1I). Whereas 
overexpressed FKBP4 resulted in downregulation of TMEM173 at mRNA level in T47D and BT549 cells (Figure 1J).

To further determine the effect of FKBP4 on TMEM173 transcriptional repression, we constructed TMEM173 promoter luciferase reporter plasmid. As shown in Figure $1 \mathrm{~K}, \mathrm{~L}$, siRNA specifically targeting FKBP4 enhanced TMEM173 promoter activity in T47D and BT549 cells, and FKBP4-HA plasmid inhibited TMEM173 promoter activity in T47D and BT549 cells, therefore FKBP4 was firstly found to have an impact on regulating TMEM173 promoter.

\subsection{Negative correlation of FKBP4 and NR3C1 in breast cancer}

We then continued to figure out potential factors involved in the FKBP4/TMEM173 axis. Firstly, we used $\mathrm{PROMO}[38,39]$, a virtual laboratory for the identification of putative transcription factors (TFs) binding sites in DNA sequences, to find predicted TFs binding to TMEM173 promoter. After inputting TMEM173 promoter sequence including 1000 bases upstream and 150 bases downstream in PROMO (Table 1), 15 TFs were shown in order of frequency: CEBPB, YY1, ERAL1, NR3C1, TBP, IRF2, FOXA1, FOXP3, STAT4, XBP1, RXRA, PAX5, TP53, GTF2I, GCFC2 (Figure 2A). Besides FKBP4 was shown to connect to NR3C1 with a higher score than FOXA1 in protein to protein interacting network (PPI) on STRING database[40] (Figure 2B) (Table 2), we wondered whether NR3C1 was involved in FKBP4 associated TMEM173 dysregulation.

We firstly used siRNA specifically targeting FKBP4 in T47D and BT549 cells, and found it led to upregulation of NR3C1 at protein level (Figure 2C). In addition, we transfected FKBP4-HA plasmid in T47D and BT549 cells, which resulted in downregulation of endogenous NR3C1 (Figure 2D). These results clearly indicated that FKBP4 had a role in negatively regulating NR3C1 protein expression. Bioinformatics results also suggested a significant negative association between FKBP4 and NR3C1 both in luminal A and basal-like subtype of BC patients (Figure S3A, B). Furthermore, we found naringenin could upregulate NR3C1 protein expression in T47D and BT549 cells (Figure S4).

\subsection{NR3C1 positively regulates TMEM173 at protein, mRNA and transcription level}

We further confirmed the specific interaction between NR3C1 and TMEM173 at the molecular level. We firstly used siRNA specifically targeting NR3C1 in T47D and BT549 cells, which led to downregulation of TMEM173 at protein level (Figure 2E). In addition, we transfected NR3C1-HA plasmid in T47D and BT549 cells, which resulted in upregulation of endogenous TMEM173 (Figure 2F). These results clearly indicated that NR3C1 had a role in positively regulating TMEM173 protein expression. Given NR3C1 was demonstrated to regulate TMEM173 at protein level, we wondered whether it affected the mRNA level of TMEM173. We then found silencing of NR3C1 led to downregulation of TMEM173 at mRNA level in T47D 
and BT549 cells (Figure 2G). Whereas overexpressed NR3C1 resulted in upregulation of TMEM173 at mRNA level in T47D and BT549 cells (Figure 2H).

At the transcriptional level, siRNA specifically targeting NR3C1 was found to decrease TMEM173 promoter activity in T47D and BT549 cells, while NR3C1-HA plasmid promoted TMEM173 promoter activity in T47D and BT549 cells (Figure $2 \mathrm{I}, \mathrm{J}$ ), therefore NR3C 1 was found to have a positive impact on regulating TMEM173 promoter.

Meanwhile, we verified a significant positive association between NR3C1 and TMEM173 both in luminal A and basal-like subtype of BC patients (Figure S3C, D). Downregulated NR3C1 was significantly related to four molecular subtype patients than the normal group (Figure S5), also strongly correlated with worse overall survival both in luminal A and basal-like subtype of BC patients (Figure S6).

Besides, knockdown of NR3C1 partially prevented siRNA targeting FKBP4 to upregulate TMEM173 expression at protein, mRNA and transcription level in BT549 and T47D cells (Figure 2K, L, M). Hence, our results implied that FKBP4 might downregulate TMEM173 by inhibiting NR3C1.

\subsection{FKBP4 binds to NR3C1 and regulates nuclear translocation of NR3C1}

As FKBP4 regulates its cellular targets via protein-protein interaction[41, 42], we tested whether FKBP4 bound to NR3C1. We performed co-IP and western blot assays using BT549 and T47D cells, and results showed that FKBP4 and NR3C1 bound with each other (Figure 3A, B). We further observed that nuclear accumulations of NR3C1 were enhanced by siRNA targeting FKBP4 or inhibited by FKBP4-HA plasmid in BT549 and T47D cells by western blot analysis (Figure 3C, D). Silencing FKBP4 mediated promotion of NR3C1 nuclear accumulations were also observed in BT549 and T47D cells by immunofluorescence microscopy (Figure 3E, F).

These observations were consistent with the finding that FKBP4 transcriptionally inhibited TMEM173 through interacting with NR3C1.

\subsection{FKBP4/NR3C1/TMEM173 signaling pathway involved in naringenin-induced autophagy}

Since naringenin had been used to enhance autophagy[43], we doubted whether naringenin related FKBP4/NR3C1/TMEM173 axis was also involved in autophagy. Western blotting showed that silencing FKBP4 decreased the expression of autophagy associated molecule P62 and increased the expression of Beclin-1 and LC3B-II/LC3B-I in T47D and BT549 cells (Figure 4A). Similarly, TMEM173 overexpression in T47D and BT549 cells led to the downregulation of P62 and upregulation of Beclin-1 and LC3B-II/LC3B-I (Figure 4B). Meanwhile, western blotting showed that FKBP4 overexpression increased the expression of 
P62 and decreased the expression of Beclin-1 and LC3B-II/LC3B-I in T47D and BT549 cells (Figure 4C), and silencing TMEM173 led to the upregulation of P62 and downregulation of Beclin-1 and LC3B-II/LC3B-I (Figure 4D). As shown in Figure S7, NR3C1 had the same effects as TMEM173 on P62, Beclin-1 and LC3B-II/LC3B-I. Autophagy flux was also observed decreased in T47D and BT549 cells silencing TMEM173 (Figure 4G, H). Furthermore, knockdown of TMEM173 significantly prevented naringenininduced promotion of T47D and BT549 cell autophagy (Figure 4E, F).

All together, these results suggested that naringenin enhanced $B C$ cell autophagy partially owing to FKBP4/NR3C1/TMEM173 axis.

\subsection{FKBP4/NR3C1/TMEM173 signaling pathway involved in naringenin-restrained cell proliferation}

Naringenin was well-known to inhibit cell proliferation[44], we doubted whether naringenin related FKBP4/NR3C1/TMEM173 axis was also involved in cell proliferation. Cell viability assay showed that silencing FKBP4 or overexpressing TMEM173 prevented cell proliferation of both T47D and BT549 cells at $72 \mathrm{~h}$ (Figure 5A, B), while overexpressing FKBP4 or silencing TMEM173 promoted cell proliferation of both T47D and BT549 cells at $72 \mathrm{~h}$ (Figure 5C, D). Similarly, we observed that silencing NR3C1 promoted cell proliferation and overexpressing NR3C1 prevented cell proliferation of both T47D and BT549 cells at $72 \mathrm{~h}$ (Figure S8). Additionally, knockdown of TMEM173 significantly prevented naringenin-restrained cell proliferation of T47D and BT549 cells during $72 \mathrm{~h}$ (Figure 5E, F), these findings were further confirmed by cell photography (Figure S9) and colony formation assay (Figure S10).

T47D cells treated with silenced TMEM173 were used to generate subcutaneous xenograft models in nude mice. Results showed that the volume and weight of tumors were significantly reduced in the naringenin treated group than in the control group, whereas those in the naringenin+shTMEM173 group were significantly greater than in the naringenin treated group (Figure $5 \mathrm{G}, \mathrm{H}, \mathrm{I}$ ). Immunohistochemical analysis showed that the staining intensity of FKBP4 was significantly lower in subcutaneous tumors in the naringenin treated group, and the expression of TMEM173 was higher than that in the control group (Figure 5J).

Thus, in vivo and in vitro experiments indicated that naringenin inhibited BC proliferation partially owing to its function of increasing TMEM173 expression.

\subsection{Exosomes secreted by naringenin treated breast cancer cells induced DC maturation}

Exosomes secreted by BC cells had been reported as cell-to-cell mediators of oncogenic or anti-cancer information[45], and further regulated DC maturation[32], we wondered whether naringenin was also involved in exosome secretion among tumor environment. After $72 \mathrm{~h}$ naringenin treatment, exosomes were 
isolated from the supernatants of $\mathrm{BC}$ cells using a two-step procedure to achieve a high purity and their morphology verified by Nanoparticle tracking analysis (Figure 6A). Next, purified exosomes derived from T47D and BT549 were further authenticated by the expression of exosome biomarkers (Alix, TSG101, CD81 and CD63) (Figure 6B). The results of Nanoparticle tracking analysis showed that the isolated exosomes had an average size of $100 \mathrm{~nm}$ (Figure 6C). Exosomes secreted by various cancer cells had been found to carry RNA and proteins, both western blot assays and RT-qPCR showed that the expression levels of NR3C1 and TMEM173 were higher in naringenin treated T47D exosomes (T47D-ex) and BT549 exosomes (BT549-ex) than in the control group, whereas FKBP4 had the opposite expression (Figure 6D, E).

We next tested the effect of exosomes secreted by naringenin treated $B C$ cells on $D C$ maturation. To this end, primary $C D 11 c^{+}$DCs were isolated from the bone marrow of mice and cultured with exosomes for 72 h. DCs cultured with naringenin treated T47D-ex and BT549-ex showed significantly increased cell surface expression of co-stimulatory molecules CD40, CD80 and CD86 (Figure 6F). We also observed the levels of IL-6, IL-12, TNF-a, IL-18, IL-1 $\beta$ and MCP-1, which reflected the maturation of DCs, were significantly upregulated by T47D-ex and BT549-ex treated with naringenin in the culture supernatant compared with those in the control group (Figure 6G-L). Thus, T47D-ex and BT549-ex exhibited adjuvant properties of promoting DC maturation.

\subsection{FKBP4/NR3C1/TMEM173 signaling pathway involved in naringenin-induced TIDC maturation}

To confirm FKBP4/NR3C1/TMEM173 axis was also regulated in DC, we co-cultured BC cells and bone marrow cells of mice with GMCSF+IL4 or naringenin, simulated as TIDC (Figure 7A). After GMCSF+IL4 treatment for $72 \mathrm{~h}$, we found that FKBP4 was gradually decreased while NR3C1 and TMEM173 were gradually increased during TIDC differentiation and maturation at both protein and mRNA level (Figure 7B, C). After naringenin treatment for $72 \mathrm{~h}$, we found that FKBP4 was gradually decreased while NR3C1 and TMEM173 were gradually increased according to naringenin concentration at both protein and mRNA level (Figure 7D, E). Flow cytometry analysis results showed that the positive expression percentages of CD11C and CD86 of TIDC were significantly increased in the naringenin group in contrast to those in the control group (Figure 7F). To demonstrate that TMEM173 truly affected the maturation and function of TIDC, we observed the levels of IL-6, IL-12, TNF-a, IL-18, IL-1 $\beta$ and MCP-1 were significantly upregulated by naringenin stimulation in the TIDC culture supernatant compared with those in the control group, opposite results were observed in the groups receiving anti-TMEM173 stimulation (Figure 7G-L). These data suggested that FKBP4/NR3C1/TMEM173 signaling pathway involved in naringenin-induced TIDC maturation.

Taken together, we demonstrated that naringenin mediated pro-autophagy, anti-proliferation of $\mathrm{BC}$ cells and pro-TIDC maturation in a new-found FKBP4/NR3C1/TMEM173 dependent way (Figure 8). 


\section{Discussion}

Recently, endogenous host-derived regulators of TMEM173 have become the hotspot on related researches. For instance, an inflammasome protein called NOD-like receptor family CARD domain containing 3 (NLRC3) blocked the direct trafficking of TMEM173 to punctuated endoplasmic-associated puncta region by direct binding to TMEM173[46]. The autophagy protein P62 negatively regulated the TMEM173 signaling by initiating the ubiquitination of TMEM173 to autophagosome[47]. As an ER membrane-bound E3-ubiquitin ligase, the RING finger protein 5 degraded TMEM173 by mediating its K48linked polyubiquitination[48]. Unlike the above-mentioned factors inhibiting TMEM173 at protein level, the current study suggested FKBP4/NR3C1 axis might be a novel endogenous negative regulator of TMEM173 by altering its transcriptional activity in both BC cells and TIDCs. Thus, FKBP4 and NR3C1 could serve as notable therapeutic molecules against TMEM173-dependent tumorigenesis, autoinflammation and autoimmunity in the future.

FK506-binding protein family in human genomes has included 18 FKBPs up to date, which could target on various pathways in embryonic development, stress response, cardiac function, cancer tumorigenesis and neuronal function[49]. In colorectal cancer, silencing FKBP3 has been found to attenuate oxaliplatin resistance by regulation of the phosphatase and tensin homolog/AKT axis[50]. In Alzheimer's disease, FKBP12 and amyloid precursor protein interplay has suspected to affect $A \beta$ peptides expression[51]. Although FKBP4 has been demonstrated to connect mammalian target of naringenin complex 2 and phosphoinositide-3-kinase to enhance cell proliferation of BC, for the first time, we found that FKBP4 played a carcinogenic role by downregulating TMEM173 in BC cells and co-cultured DCs.

Currently, there is no research of regulation mechanisms on NR3C1 and cGAS-STING pathway, but only a few studies suggest that NR3C1 is involved in the innate immune response[52, 53]. In our study, we demonstrated FKBP4 transcriptionally downregulated TMEM173 through binding to NR3C1, thus inhibiting nuclear translocation of NR3C1, but this effect was found relatively weak because shNR3C1 could not completely inhibit FKBP4 from regulating TMEM173 according to results of Fig. 2K. Therefore, we speculated that some of the predicted TFs were also involved in FKBP4/TMEM173 pathway, subsequent mechanism studies need to be further improved to verify our conjecture.

As for the interaction of FKBP and autophagy, FKBP5 has been reported to change phosphorylation of Beclin1 by binding to Beclin1, thus triggering autophagic pathways[54]. FKBP8 was found to recruit lipidated LC3A to induce mitochondrial autophagy via N-terminal LC3-interacting region motif of FKBP8[55]. While the regulation of autophagy by FKBP4 in tumor cells has not been reported yet. Meanwhile, TMEM173 could activate autophagy through inducing LC3B lipidation, which was independent of TANK binding kinase 1 activation and interferon induction[56]. In current study, we firstly demonstrated that inhibiting FKBP4 could promote autophagy by increasing TMEM173 expression of BC cells.

TMEM173 expression has been reported suppressed or lost in majority of cancers, especially in BC[5759], which suggests that during $B C$ progression, downregulation of the proteins involved in innate immune response may be helpful for evading innate immune response pathways to facilitate tumor growth. 
Although hypermethylation of the promoter regions in TMEM173 has been revealed in colorectal cancer[15], little is known about the mechanism of decreased TMEM173 expression in BC. Our results imply FKBP4 could induce TMEM173 transcriptional suppression via NR3C1, it would be interesting to further study the correlation between TMEM173 and other FKBPs involved in innate immune response and relevance during breast tumorigenesis.

Demaria et al. demonstrated that IFN-stimulatory dsDNA from irradiated BC cells, which are phagocytosed by DCs via stimulating cGAS/TMEM173 pathway[32]. Thus, it is likely that different methods mediate the transfer of other cargos to DCs through TMEM173 related signalings, e.g., our study firstly found that exosomes secreted by naringenin treated BC cells induced DC maturation on TMEM173 dependent way. Further experimentation will be required to determine the relative contribution of these different mechanisms of tumor DNA delivery to DCs.

Recently, a growing body of evidence has demonstrated that the pharmacological agents, e.g., dimethyloxoxanthenyl acetic acid, 2'3'-cGAMP, ML RR-S2 cGAMP, ML RR-S2 CDA could induce activation of TMEM173[60-63]. Here we proposed naringenin, a citrus flavonoid shown to have cytotoxic and antiproliferative effects on various cancer cell types[64] and regulate immunological pathways[65], to be another kind of potential TMEM173 conditioning agent. With the development of immunotherapies such as cancer vaccine, immune checkpoint inhibitors, oncolytic virus, and chimeric antigen receptor $\mathrm{T}$ cell therapy[66], combination of TMEM173-targeting agonists and immunotherapies may provide multiple feasible approaches to new BC treatment strategies.

\section{Conclusions}

In summary, we demonstrated that FKBP4 transcriptionally downregulated TMEM173 through inhibiting nuclear translocation of $\mathrm{NR3C1}$, naringenin promoted autophagy, suppressed proliferation in luminal A and basal-like subtype of $B C$ cells and enhanced TIDC maturation via new-found FKBP4/NR3C1/TMEM173 axis. We proposed that FKBP4/NR3C1/TMEM173 signaling pathway served as a potential immunological therapeutic target for the treatment of $\mathrm{BC}$ patients.

\section{Abbreviations}

BC breast cancer

TIDC tumor-infiltrating dendritic cell

FKBP FK506-binding protein

TMEM173 transmembrane protein 173

STING stimulator of interferon genes

cGAS cyclic GMP-AMP synthase

Page 15/38 
NR3C1 nuclear receptor subfamily 3 , group C, member 1

Ex exosome

TF transcription factor

PPI protein-protein interaction

\section{Declarations}

\section{ETHICS APPROVAL AND CONSENT TO PARTICIPATE}

Not applicable.

\section{CONSENT FOR PUBLICATION}

Not applicable.

\section{AVAILABILITY OF DATA AND MATERIAL}

The datasets used and/or analyzed during the current study are available from the corresponding author on reasonable request.

\section{COMPETING INTERESTS}

The authors declare that they have no competing interests.

\section{FUNDING}

The work was supported by the National Natural Science Foundation of China (No. 81972453, No. 81972597, No. 81602471, No. 81672729 and No. 82000212), Zhejiang Provincial Natural Science Foundation of China under Grants (No. LY19H160055, LY19H160059, LY18H160005, LY18H160030, LY20H160026 and LQ21H160022). The work was sponsored by Zhejiang Provincial Medical and Health Science and Technology Project (No. 2018ZD028 and No. 2021RC003).

\section{AUTHORS' CONTRIBUTIONS}

$\mathrm{XHC}$ designed the experiment. $\mathrm{XHC}$ and $\mathrm{CZH}$ performed most of experiments. $\mathrm{LBH}, \mathrm{CC}$ and $\mathrm{LZQ}$ performed the western blot assay and the real-time PCR experiments. JYS contributed to bioinformatics analysis. $\mathrm{XHC}, \mathrm{CZH}$ and $\mathrm{LBH}$ analyzed the data and wrote the paper. WLB and ZJC conducted the study supervision. All authors read and approved the final manuscript.

\section{ACKNOWLEDGMENTS}

Thanks for the technical support by the Core Facilities, Department of Radiation Oncology, Zhejiang Provincial People's Hospital. 


\section{References}

1. Ganz PA, Goodwin PJ: Breast Cancer Survivorship: Where Are We Today? Adv Exp Med Biol 2015, 862:1-8.

2. Yu LY, Tang J, Zhang CM, Zeng WJ, Yan H, Li MP, Chen XP: New Immunotherapy Strategies in Breast Cancer. International journal of environmental research and public health 2017, 14(1).

3. Vonderheide RH, LoRusso PM, Khalil M, Gartner EM, Khaira D, Soulieres D, Dorazio P, Trosko JA, Rüter $\mathrm{J}$, Mariani GL et al: Tremelimumab in combination with exemestane in patients with advanced breast cancer and treatment-associated modulation of inducible costimulator expression on patient $\mathrm{T}$ cells. Clin Cancer Res 2010, 16(13):3485-3494.

4. Lum LG, Thakur A, Al-Kadhimi Z, Colvin GA, Cummings FJ, Legare RD, Dizon DS, Kouttab N, Maizel A, Colaiace W et al: Targeted T-cell Therapy in Stage IV Breast Cancer: A Phase I Clinical Trial. Clin Cancer Res 2015, 21(10):2305-2314.

5. De luliis F, Salerno G, Taglieri L, Scarpa S: Are pharmacogenomic biomarkers an effective tool to predict taxane toxicity and outcome in breast cancer patients? Literature review. Cancer chemotherapy and pharmacology 2015, 76(4):679-690.

6. Ishikawa H, Barber GN: STING is an endoplasmic reticulum adaptor that facilitates innate immune signalling. Nature 2008, 455(7213):674-678.

7. Sun L, Wu J, Du F, Chen X, Chen ZJ: Cyclic GMP-AMP synthase is a cytosolic DNA sensor that activates the type I interferon pathway. Science 2013, 339(6121):786-791.

8. Wu J, Sun L, Chen X, Du F, Shi H, Chen C, Chen ZJ: Cyclic GMP-AMP is an endogenous second messenger in innate immune signaling by cytosolic DNA. Science 2013, 339(6121):826-830.

9. Holm CK, Jensen SB, Jakobsen MR, Cheshenko N, Horan KA, Moeller HB, Gonzalez-Dosal R, Rasmussen SB, Christensen MH, Yarovinsky TO et al: Virus-cell fusion as a trigger of innate immunity dependent on the adaptor STING. Nat Immunol 2012, 13(8):737-743.

10. Ahn J, Gutman D, Saijo S, Barber GN: STING manifests self DNA-dependent inflammatory disease. Proceedings of the National Academy of Sciences of the United States of America 2012, 109(47):19386-19391.

11. Pantelidou C, Sonzogni O, De Oliveria Taveira M, Mehta AK, Kothari A, Wang D, Visal T, Li MK, Pinto J, Castrillon JA et al: PARP Inhibitor Efficacy Depends on CD8(+) T-cell Recruitment via Intratumoral STING Pathway Activation in BRCA-Deficient Models of Triple-Negative Breast Cancer. Cancer discovery 2019.

12. Song S, Peng P, Tang Z, Zhao J, Wu W, Li H, Shao M, Li L, Yang C, Duan F et al: Decreased expression of STING predicts poor prognosis in patients with gastric cancer. Scientific reports 2017, 7:39858.

13. Tang CH, Zundell JA, Ranatunga S, Lin C, Nefedova Y, Del Valle JR, Hu CC: Agonist-Mediated Activation of STING Induces Apoptosis in Malignant B Cells. Cancer Res 2016, 76(8):2137-2152.

14. Ho SS, Zhang WY, Tan NY, Khatoo M, Suter MA, Tripathi S, Cheung FS, Lim WK, Tan PH, Ngeow J et al: The DNA Structure-Specific Endonuclease MUS81 Mediates DNA Sensor STING-Dependent Host 
Rejection of Prostate Cancer Cells. Immunity 2016, 44(5):1177-1189.

15. Xia T, Konno H, Ahn J, Barber GN: Deregulation of STING Signaling in Colorectal Carcinoma Constrains DNA Damage Responses and Correlates With Tumorigenesis. Cell reports 2016, 14(2):282297.

16. Nakamura T, Miyabe H, Hyodo M, Sato Y, Hayakawa Y, Harashima H: Liposomes loaded with a STING pathway ligand, cyclic di-GMP, enhance cancer immunotherapy against metastatic melanoma. Journal of controlled release : official journal of the Controlled Release Society 2015, 216:149-157.

17. Su J, Rui Y, Lou M, Yin L, Xiong H, Zhou Z, Shen S, Chen T, Zhang Z, Zhao N et al: HIV-2/SIV Vpx targets a novel functional domain of STING to selectively inhibit cGAS-STING-mediated NF-KB signalling. Nat Microbiol 2019, 4(12):2552-2564.

18. Federer-Gsponer JR, Quintavalle C, Muller DC, Dietsche T, Perrina V, Lorber T, Juskevicius D, Lenkiewicz $\mathrm{E}$, Zellweger T, Gasser T et al: Delineation of human prostate cancer evolution identifies chromothripsis as a polyclonal event and FKBP4 as a potential driver of castration resistance. The Journal of pathology 2018, 245(1):74-84.

19. Kiyamova R, Garifulin O, Gryshkova V, Kostianets O, Shyian M, Gout I, Filonenko V: Preliminary study of thyroid and colon cancers-associated antigens and their cognate autoantibodies as potential cancer biomarkers. Biomarkers : biochemical indicators of exposure, response, and susceptibility to chemicals 2012, 17(4):362-371.

20. Yang WS, Moon HG, Kim HS, Choi EJ, Yu MH, Noh DY, Lee C: Proteomic approach reveals FKBP4 and S100A9 as potential prediction markers of therapeutic response to neoadjuvant chemotherapy in patients with breast cancer. Journal of proteome research 2012, 11(2):1078-1088.

21. Mohanta S, Sekhar Khora S, Suresh A: Cancer Stem Cell based molecular predictors of tumor recurrence in Oral squamous cell carcinoma. Archives of oral biology 2019, 99:92-106.

22. Xiong H, Chen Z, Zheng W, Sun J, Fu Q, Teng R, Chen J, Xie S, Wang L, Yu XF et al: FKBP4 is a malignant indicator in luminal A subtype of breast cancer. $J$ Cancer 2020, 11(7):1727-1736.

23. Ebong IO, Beilsten-Edmands V, Patel NA, Morgner N, Robinson CV: The interchange of immunophilins leads to parallel pathways and different intermediates in the assembly of Hsp90 glucocorticoid receptor complexes. Cell discovery 2016, 2:16002.

24. Chambraud B, Radanyi C, Camonis JH, Rajkowski K, Schumacher M, Baulieu EE: Immunophilins, Refsum disease, and lupus nephritis: the peroxisomal enzyme phytanoyl-COA alpha-hydroxylase is a new FKBP-associated protein. Proceedings of the National Academy of Sciences of the United States of America 1999, 96(5):2104-2109.

25. Xiong $\mathrm{H}$, Chen Z, Chen W, Li Q, Lin B, Jia Y: FKBP-related ncRNA-mRNA axis in breast cancer. Genomics 2020, 112(6):4595-4607.

26. Argentieri MA, Nagarajan S, Seddighzadeh B, Baccarelli AA, Shields AE: Epigenetic Pathways in Human Disease: The Impact of DNA Methylation on Stress-Related Pathogenesis and Current Challenges in Biomarker Development. EBioMedicine 2017, 18:327-350. 
27. Vilasco M, Communal L, Mourra N, Courtin A, Forgez P, Gompel A: Glucocorticoid receptor and breast cancer. Breast cancer research and treatment 2011, 130(1):1-10.

28. Ruiz-Conca M, Gardela J, Martínez CA, Wright D, López-Bejar M, Rodríguez-Martínez H, ÁlvarezRodríguez M: Natural Mating Differentially Triggers Expression of Glucocorticoid Receptor (NR3C1)Related Genes in the Preovulatory Porcine Female Reproductive Tract. International journal of molecular sciences 2020, 21(12).

29. Vader P, Breakefield XO, Wood MJ: Extracellular vesicles: emerging targets for cancer therapy. Trends in molecular medicine 2014, 20(7):385-393.

30. Sharma A, Johnson A: Exosome DNA: Critical regulator of tumor immunity and a diagnostic biomarker. Journal of cellular physiology 2020, 235(3):1921-1932.

31. Wen SW, Sceneay J, Lima LG, Wong CS, Becker M, Krumeich S, Lobb RJ, Castillo V, Wong KN, Ellis S et al: The Biodistribution and Immune Suppressive Effects of Breast Cancer-Derived Exosomes. Cancer Res 2016, 76(23):6816-6827.

32. Diamond JM, Vanpouille-Box C, Spada S, Rudqvist NP, Chapman JR, Ueberheide BM, Pilones KA, Sarfraz Y, Formenti SC, Demaria S: Exosomes Shuttle TREX1-Sensitive IFN-Stimulatory dsDNA from Irradiated Cancer Cells to DCs. Cancer immunology research 2018, 6(8):910-920.

33. Salehi B, Fokou PVT, Sharifi-Rad M, Zucca P, Pezzani R, Martins N, Sharifi-Rad J: The Therapeutic Potential of Naringenin: A Review of Clinical Trials. Pharmaceuticals (Basel, Switzerland) 2019, 12(1).

34. Zhang Z, Zhou C, Li X, Barnes SD, Deng S, Hoover E, Chen CC, Lee YS, Zhang Y, Wang C et al: Loss of CHD1 Promotes Heterogeneous Mechanisms of Resistance to AR-Targeted Therapy via Chromatin Dysregulation. Cancer cell 2020, 37(4):584-598.e511.

35. Zhou Z, Chen H, Xie R, Wang H, Li S, Xu Q, Xu N, Cheng Q, Qian Y, Huang R et al: Epigenetically modulated FOXM1 suppresses dendritic cell maturation in pancreatic cancer and colon cancer. $\mathrm{Mol}$ Oncol 2019, 13(4):873-893.

36. Jézéquel P, Campone M, Gouraud W, Guérin-Charbonnel C, Leux C, Ricolleau G, Campion L: bcGenExMiner: an easy-to-use online platform for gene prognostic analyses in breast cancer. Breast cancer research and treatment 2012, 131(3):765-775.

37. Zhang L, He H, Zhang M, Wu Y, Xu X, Yang M, Mei L: Assessing the effect and related mechanism of naringenin on the proliferation, osteogenic differentiation and endothelial differentiation of human periodontal ligament stem cells. Biochemical and biophysical research communications 2021, 534:337-342.

38. Messeguer X, Escudero R, Farre D, Nunez O, Martinez J, Alba MM: PROMO: detection of known transcription regulatory elements using species-tailored searches. Bioinformatics (Oxford, England) 2002, 18(2):333-334.

39. Farre D, Roset R, Huerta M, Adsuara JE, Rosello L, Alba MM, Messeguer X: Identification of patterns in biological sequences at the ALGGEN server: PROMO and MALGEN. Nucleic acids research 2003, 31(13):3651-3653. 
40. Szklarczyk D, Gable AL, Lyon D, Junge A, Wyder S, Huerta-Cepas J, Simonovic M, Doncheva NT, Morris $\mathrm{JH}$, Bork P et al: STRING v11: protein-protein association networks with increased coverage, supporting functional discovery in genome-wide experimental datasets. Nucleic acids research 2019, 47(D1):D607-d613.

41. Assimon VA, Southworth DR, Gestwicki JE: Specific Binding of Tetratricopeptide Repeat Proteins to Heat Shock Protein 70 (Hsp70) and Heat Shock Protein 90 (Hsp90) Is Regulated by Affinity and Phosphorylation. Biochemistry 2015, 54(48):7120-7131.

42. Kimmins S, MacRae TH: Maturation of steroid receptors: an example of functional cooperation among molecular chaperones and their associated proteins. Cell stress \& chaperones 2000, 5(2):7686.

43. Ahsan AU, Sharma VL, Wani A, Chopra M: Naringenin Upregulates AMPK-Mediated Autophagy to Rescue Neuronal Cells From $\beta$-Amyloid ((1-42)) Evoked Neurotoxicity. Mol Neurobiol 2020, 57(8):3589-3602.

44. Pateliya B, Burade V, Goswami S: Combining naringenin and metformin with doxorubicin enhances anticancer activity against triple-negative breast cancer in vitro and in vivo. European journal of pharmacology 2021, 891:173725.

45. Melo SA, Sugimoto H, O'Connell JT, Kato N, Villanueva A, Vidal A, Qiu L, Vitkin E, Perelman LT, Melo CA et al: Cancer exosomes perform cell-independent microRNA biogenesis and promote tumorigenesis. Cancer cell 2014, 26(5):707-721.

46. Zhang L, Mo J, Swanson KV, Wen H, Petrucelli A, Gregory SM, Zhang Z, Schneider M, Jiang Y, Fitzgerald KA et al: NLRC3, a member of the NLR family of proteins, is a negative regulator of innate immune signaling induced by the DNA sensor STING. Immunity 2014, 40(3):329-341.

47. Prabakaran T, Bodda C, Krapp C, Zhang BC, Christensen MH, Sun C, Reinert L, Cai Y, Jensen SB, Skouboe MK et al: Attenuation of cGAS-STING signaling is mediated by a p62/SQSTM1-dependent autophagy pathway activated by TBK1. The EMBO journal 2018, 37(8).

48. Qin Y, Zhou MT, Hu MM, Hu YH, Zhang J, Guo L, Zhong B, Shu HB: RNF26 temporally regulates virustriggered type I interferon induction by two distinct mechanisms. PLOS pathogens 2014, 10(9):e1004358.

49. Ghartey-Kwansah G, Li Z, Feng R, Wang L, Zhou X, Chen FZ, Xu MM, Jones O, Mu Y, Chen S et al: Comparative analysis of FKBP family protein: evaluation, structure, and function in mammals and Drosophila melanogaster. BMC Dev Bio/ 2018, 18(1):7.

50. Tong J, Shen Y, Chen X, Wang R, Hu Y, Zhang X, Zhang Z, Han L: FKBP3 mediates oxaliplatin resistance in colorectal cancer cells by regulating HDAC2 expression. Oncology reports 2019.

51. Blair LJ, Baker JD, Sabbagh JJ, Dickey CA: The emerging role of peptidyl-prolyl isomerase chaperones in tau oligomerization, amyloid processing, and Alzheimer's disease. Journal of neurochemistry 2015, 133(1):1-13.

52. Abdelmegeid MK, Vailati-Riboni M, Alharthi A, Batistel F, Loor JJ: Supplemental methionine, choline, or taurine alter in vitro gene network expression of polymorphonuclear leukocytes from neonatal 
Holstein calves. Journal of dairy science 2017, 100(4):3155-3165.

53. Smith AJ, Li Q, Wietgrefe SW, Schacker TW, Reilly CS, Haase AT: Host genes associated with HIV-1 replication in lymphatic tissue. Journal of immunology (Baltimore, Md : 1950) 2010, 185(9):54175424.

54. Gassen NC, Hartmann J, Zschocke J, Stepan J, Hafner K, Zellner A, Kirmeier T, Kollmannsberger L, Wagner KV, Dedic $\mathrm{N}$ et al: Association of FKBP51 with priming of autophagy pathways and mediation of antidepressant treatment response: evidence in cells, mice, and humans. PLoS medicine 2014, 11(11):e1001755.

55. Bhujabal Z, Birgisdottir Å B, Sjøttem E, Brenne HB, Øvervatn A, Habisov S, Kirkin V, Lamark T, Johansen T: FKBP8 recruits LC3A to mediate Parkin-independent mitophagy. EMBO reports 2017, 18(6):947961.

56. Gui X, Yang H, Li T, Tan X, Shi P, Li M, Du F, Chen ZJ: Autophagy induction via STING trafficking is a primordial function of the cGAS pathway. Nature 2019, 567(7747):262-266.

57. Bhatelia K, Singh A, Tomar D, Singh K, Sripada L, Chagtoo M, Prajapati P, Singh R, Godbole MM, Singh R: Antiviral signaling protein MITA acts as a tumor suppressor in breast cancer by regulating NFkappaB induced cell death. Biochimica et biophysica acta 2014, 1842(2):144-153.

58. Gaston J, Cheradame L, Yvonnet V, Deas O, Poupon MF, Judde JG, Cairo S, Goffin V: Intracellular STING inactivation sensitizes breast cancer cells to genotoxic agents. Oncotarget 2016, 7(47):7720577224.

59. Parkes EE, Walker SM, Taggart LE, McCabe N, Knight LA, Wilkinson R, McCloskey KD, Buckley NE, Savage KI, Salto-Tellez M et al: Activation of STING-Dependent Innate Immune Signaling By S-PhaseSpecific DNA Damage in Breast Cancer. Journal of the National Cancer Institute 2017, 109(1).

60. Mukai K, Konno H, Akiba T, Uemura T, Waguri S, Kobayashi T, Barber GN, Arai H, Taguchi T: Activation of STING requires palmitoylation at the Golgi. Nat Commun 2016, 7:11932.

61. Marcus A, Mao AJ, Lensink-Vasan M, Wang L, Vance RE, Raulet DH: Tumor-Derived cGAMP Triggers a STING-Mediated Interferon Response in Non-tumor Cells to Activate the NK Cell Response. Immunity 2018, 49(4):754-763.e754.

62. Corrales L, Glickman LH, McWhirter SM, Kanne DB, Sivick KE, Katibah GE, Woo SR, Lemmens E, Banda T, Leong JJ et al: Direct Activation of STING in the Tumor Microenvironment Leads to Potent and Systemic Tumor Regression and Immunity. Cell reports 2015, 11(7):1018-1030.

63. Foote JB, Kok M, Leatherman JM, Armstrong TD, Marcinkowski BC, Ojalvo LS, Kanne DB, Jaffee EM, Dubensky TW, Jr., Emens LA: A STING Agonist Given with OX40 Receptor and PD-L1 Modulators Primes Immunity and Reduces Tumor Growth in Tolerized Mice. Cancer immunology research 2017, 5(6):468-479.

64. Hermawan A, Ikawati M, Jenie RI, Khumaira A, Putri H, Nurhayati IP, Angraini SM, Muflikhasari HA: Identification of potential therapeutic target of naringenin in breast cancer stem cells inhibition by bioinformatics and in vitro studies. Saudi pharmaceutical journal : SPJ : the official publication of the Saudi Pharmaceutical Society 2021, 29(1):12-26. 
65. Zhong J, Yu R, Zhou Q, Liu P, Liu Z, Bian Y: Naringenin prevents TNF-a-induced gut-vascular barrier disruption associated with inhibiting the NF-KB-mediated MLCK/p-MLC and NLRP3 pathways. Food \& function 2021, 12(6):2715-2725.

66. Li A, Yi M, Qin S, Song Y, Chu Q, Wu K: Activating cGAS-STING pathway for the optimal effect of cancer immunotherapy. J Hematol Oncol 2019, 12(1):35.

\section{Tables}

Table 1 TMEM173 promoter sequence including 1000 bases upstream and 150 bases downstream.

gcagtggtgccatcacaactcactgcaacctctacctccaggggtcaagtgatgctcccacctcagcctcccaag tagctgggactataggcgtgttccgtcatgcttggctaattttttttttttttttgtagagatgggatctccctgtgttgctta ggctggtctcaaacttctgggctcaagtgatcctcctgccttggcctcccaaagtgctgggattactggaatgaaatca aggcacagagcaagctgggctttggagcaacccaccaggcttcaagtccccactctcaattacttaaaccagttattt cacctccctgagcctcggattatccatctataaaatggggctagaattatacctacctgacagggtggctggtgaaatgat atacaagtgaagtgatatatgcaacacttggcataatgtctggaacaaggtaaacactttattattattattattattataatttagg ttgatgcatggggattttataacctacactcaaacaatgtaggtcagatcatttttcttttcttttctttcttttcttttga gacagtctcgctcttgttgcccaggctggagtgcagtggcctaatctctgctcactgcaacttccacctctcaggttccagcgattc tcctgcctcagcctcccaagtagctaagattacaagcgcccaccaccacgcctggctaatttttgttttagtagagatggggt ttcaccatgttgctcaggctggtcttgaactcctgacctcaagtgatccacccatctcggcctcccaaagcgctgggattacaggcatgagcca ctgtgccaggcctgcaattacttttgctcctacctaatatcatccccacaaccgccttctgggcagaaaccggcagg ctctcttggagaagtcacag gcgtggccatttcctgcaaagagccaaacccccattcctctgtgcccctcctctcccaccaagtgcttTATAAAAATAGC TCTTGTTACCGGAAATAACTGTTCATTTTTCACTCCTCCCTCCTAGGTCACACTTTT CAGAAAAAGAATCTGCATCCTGGAAACCAGAAGAAAAATATGAGACGGGGAATCATCGTG TGATGTGTGTGCTGCCTTTGG

Table 2 Protein to protein interacting score of FKBP4 and predicted TMEM173 transcription factors. 


\begin{tabular}{|c|c|c|c|c|}
\hline node1 & node2 & node1 accession & node2 accession & score \\
\hline YY1 & TP53 & ENSP00000262238 & ENSP00000269305 & 0.987 \\
\hline TP53 & YY1 & ENSP00000269305 & ENSP00000262238 & 0.987 \\
\hline TP53 & TBP & ENSP00000269305 & ENSP00000375942 & 0.986 \\
\hline TBP & TP53 & ENSP00000375942 & ENSP00000269305 & 0.986 \\
\hline NR3C1 & FKBP4 & ENSP00000231509 & ENSP00000001008 & 0.982 \\
\hline FKBP4 & NR3C1 & ENSP00000001008 & ENSP00000231509 & 0.982 \\
\hline TP53 & NR3C1 & ENSP00000269305 & ENSP00000231509 & 0.971 \\
\hline NR3C1 & TP53 & ENSP00000231509 & ENSP00000269305 & 0.971 \\
\hline TBP & FOXA1 & ENSP00000375942 & ENSP00000250448 & 0.953 \\
\hline FOXA1 & TBP & ENSP00000250448 & ENSP00000375942 & 0.953 \\
\hline YY1 & FOXA1 & ENSP00000262238 & ENSP00000250448 & 0.947 \\
\hline FOXA1 & YY1 & ENSP00000250448 & ENSP00000262238 & 0.947 \\
\hline RXRA & CEBPB & ENSP00000419692 & ENSP00000305422 & 0.946 \\
\hline CEBPB & RXRA & ENSP00000305422 & ENSP00000419692 & 0.946 \\
\hline YY1 & RXRA & ENSP00000262238 & ENSP00000419692 & 0.944 \\
\hline RXRA & YY1 & ENSP00000419692 & ENSP00000262238 & 0.944 \\
\hline RXRA & NR3C1 & ENSP00000419692 & ENSP00000231509 & 0.923 \\
\hline NR3C1 & RXRA & ENSP00000231509 & ENSP00000419692 & 0.923 \\
\hline NR3C1 & IRF2 & ENSP00000231509 & ENSP00000377218 & 0.917 \\
\hline IRF2 & NR3C1 & ENSP00000377218 & ENSP00000231509 & 0.917 \\
\hline FOXA1 & FKBP4 & ENSP00000250448 & ENSP00000001008 & 0.913 \\
\hline FKBP4 & FOXA1 & ENSP00000001008 & ENSP00000250448 & 0.913 \\
\hline YY1 & GTF2I & ENSP00000262238 & ENSP00000460070 & 0.722 \\
\hline GTF2I & YY1 & ENSP00000460070 & ENSP00000262238 & 0.722 \\
\hline NR3C1 & CEBPB & ENSP00000231509 & ENSP00000305422 & 0.683 \\
\hline CEBPB & NR3C1 & ENSP00000305422 & ENSP00000231509 & 0.683 \\
\hline XBP1 & PAX5 & ENSP00000216037 & ENSP00000350844 & 0.654 \\
\hline PAX5 & XBP1 & ENSP00000350844 & ENSP00000216037 & 0.654 \\
\hline
\end{tabular}




\begin{tabular}{|c|c|c|c|c|}
\hline TP53 & CEBPB & ENSP00000269305 & ENSP00000305422 & 0.643 \\
\hline CEBPB & TP53 & ENSP00000305422 & ENSP00000269305 & 0.643 \\
\hline YY1 & TBP & ENSP00000262238 & ENSP00000375942 & 0.618 \\
\hline TBP & YY1 & ENSP00000375942 & ENSP00000262238 & 0.618 \\
\hline STAT4 & FOXP3 & ENSP00000376134 & ENSP00000365380 & 0.601 \\
\hline FOXP3 & STAT4 & ENSP00000365380 & ENSP00000376134 & 0.601 \\
\hline TP53 & PAX5 & ENSP00000269305 & ENSP00000350844 & 0.583 \\
\hline PAX5 & TP53 & ENSP00000350844 & ENSP00000269305 & 0.583 \\
\hline TBP & PAX5 & ENSP00000375942 & ENSP00000350844 & 0.58 \\
\hline PAX5 & TBP & ENSP00000350844 & ENSP00000375942 & 0.58 \\
\hline TBP & NR3C1 & ENSP00000375942 & ENSP00000231509 & 0.579 \\
\hline NR3C1 & TBP & ENSP00000231509 & ENSP00000375942 & 0.579 \\
\hline YY1 & FOXP3 & ENSP00000262238 & ENSP00000365380 & 0.566 \\
\hline FOXP3 & YY1 & ENSP00000365380 & ENSP00000262238 & 0.566 \\
\hline TP53 & FKBP4 & ENSP00000269305 & ENSP00000001008 & 0.561 \\
\hline FKBP4 & TP53 & ENSP00000001008 & ENSP00000269305 & 0.561 \\
\hline YY1 & CEBPB & ENSP00000262238 & ENSP00000305422 & 0.56 \\
\hline CEBPB & YY1 & ENSP00000305422 & ENSP00000262238 & 0.56 \\
\hline FOXA1 & CEBPB & ENSP00000250448 & ENSP00000305422 & 0.553 \\
\hline CEBPB & FOXA1 & ENSP00000305422 & ENSP00000250448 & 0.553 \\
\hline YY1 & PAX5 & ENSP00000262238 & ENSP00000350844 & 0.545 \\
\hline PAX5 & YY1 & ENSP00000350844 & ENSP00000262238 & 0.545 \\
\hline TP53 & FOXA1 & ENSP00000269305 & ENSP00000250448 & 0.53 \\
\hline FOXA1 & TP53 & ENSP00000250448 & ENSP00000269305 & 0.53 \\
\hline XBP1 & FOXA1 & ENSP00000216037 & ENSP00000250448 & 0.523 \\
\hline FOXA1 & XBP1 & ENSP00000250448 & ENSP00000216037 & 0.523 \\
\hline NR3C1 & FOXA1 & ENSP00000231509 & ENSP00000250448 & 0.51 \\
\hline FOXA1 & NR3C1 & ENSP00000250448 & ENSP00000231509 & 0.51 \\
\hline TBP & RXRA & ENSP00000375942 & ENSP00000419692 & 0.501 \\
\hline RXRA & TBP & ENSP00000419692 & ENSP00000375942 & 0.501 \\
\hline
\end{tabular}




\begin{tabular}{|lllll|}
\hline XBP1 & TP53 & ENSP00000216037 & ENSP00000269305 & 0.491 \\
\hline TP53 & XBP1 & ENSP00000269305 & ENSP00000216037 & 0.491 \\
\hline YY1 & NR3C1 & ENSP00000262238 & ENSP00000231509 & 0.481 \\
\hline NR3C1 & YY1 & ENSP00000231509 & ENSP00000262238 & 0.481 \\
\hline TP53 & FOXP3 & ENSP00000269305 & ENSP00000365380 & 0.464 \\
\hline FOXP3 & TP53 & ENSP00000365380 & ENSP00000269305 & 0.464 \\
\hline RXRA & FOXA1 & ENSP00000419692 & ENSP00000250448 & 0.446 \\
\hline FOXA1 & RXRA & ENSP00000250448 & ENSP00000419692 & 0.446 \\
\hline TBP & CEBPB & ENSP00000375942 & ENSP00000305422 & 0.405 \\
\hline CEBPB & TBP & ENSP00000305422 & ENSP00000375942 & 0.405 \\
\hline TBP & GTF2I & ENSP00000375942 & ENSP00000460070 & 0.403 \\
\hline GTF2I & TBP & ENSP00000460070 & ENSP00000375942 & 0.403 \\
\hline
\end{tabular}

Figures 

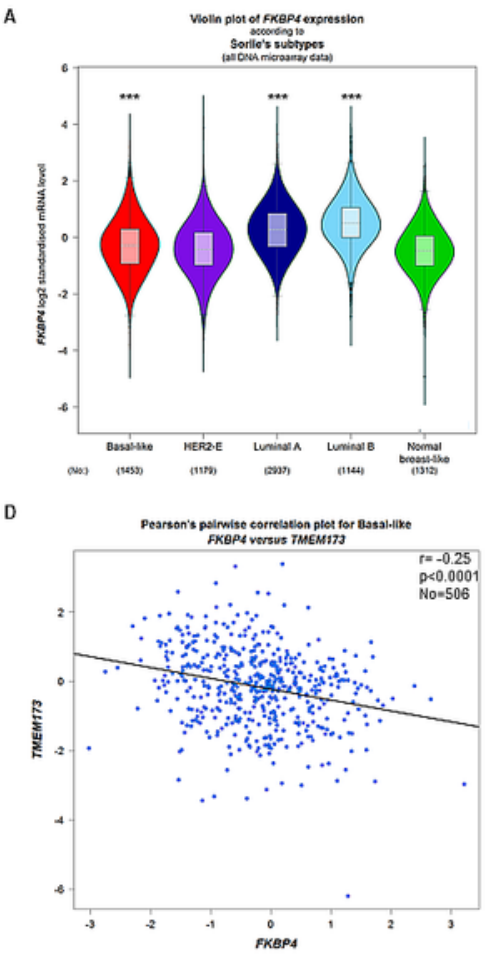

B

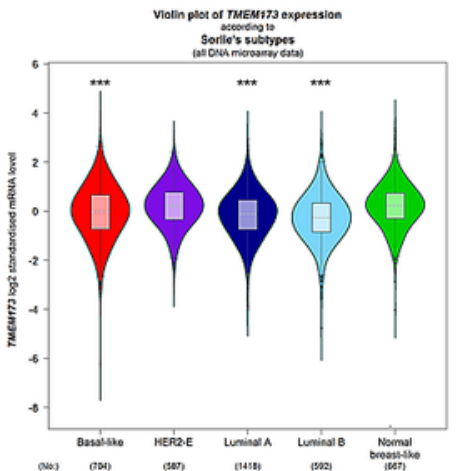

$\mathrm{E}$

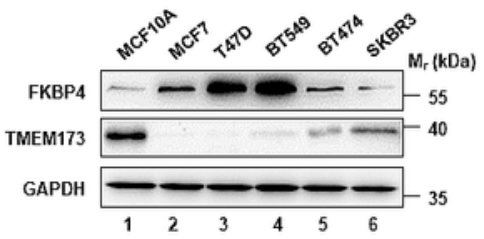

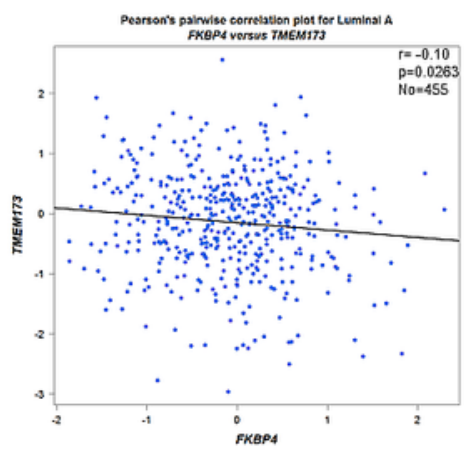

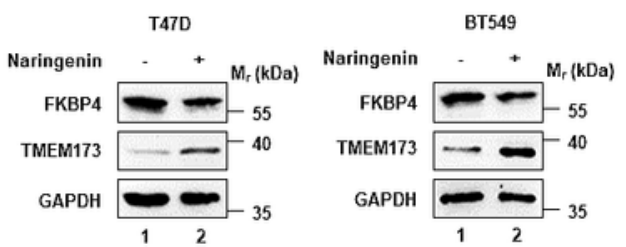

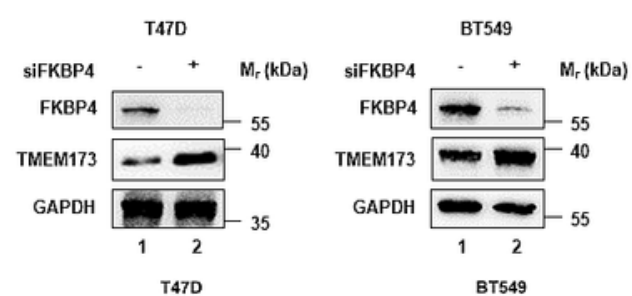

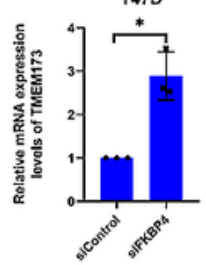

K
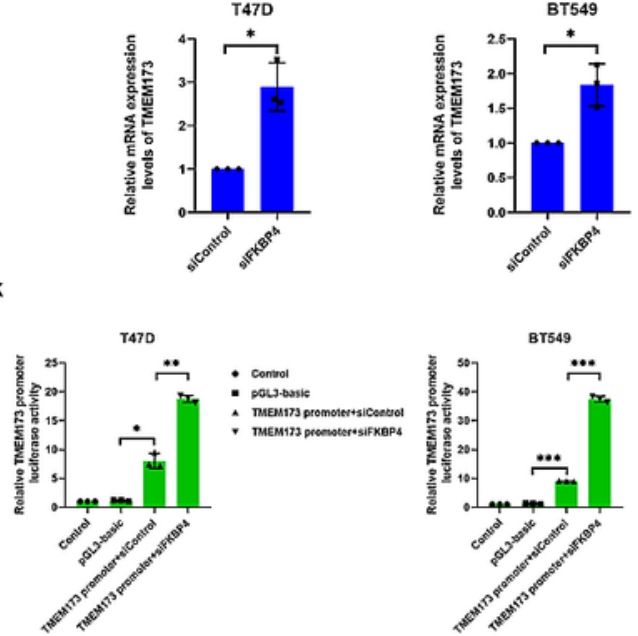
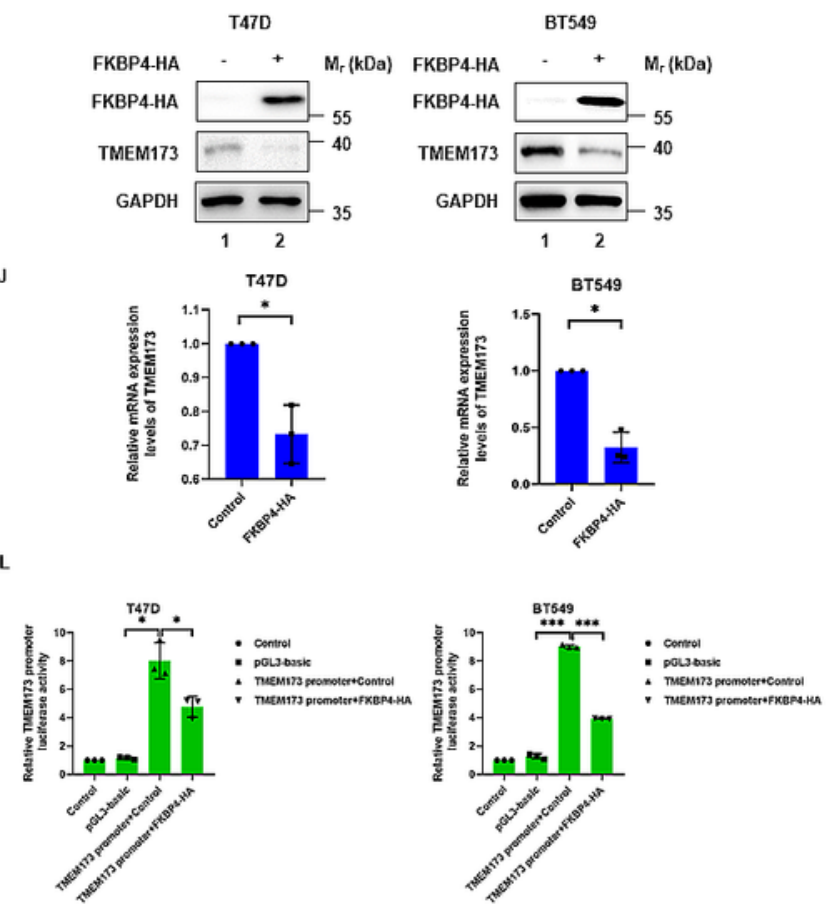

Figure 1

FKBP4 negatively regulates TMEM173 at protein, mRNA and transcription level. a A violin plot indicated upregulated FKBP4 in luminal $A$, luminal $B$ and basal-like subtype of $B C$ patients than the normal group. $b$ A violin plot indicated downregulated TMEM173 in luminal A, luminal B and basal-like subtype of BC patients than the normal group. $c$ Pearson's pairwise correlation plot of FKBP4 and TMEM173 in luminal A subtype of $B C$ patients, $r=-0.10, p=0.0263, N o=455$. $d$ Pearson's pairwise correlation plot of FKBP4 and 
TMEM173 in basal-like subtype of $B C$ patients, $r=-0.25, p<0.0001, \mathrm{No}=506$. e Representative western blot analysis results of FKBP4, TMEM173 and endogenous control GAPDH. Western blot analysis showed a negatively correlated expression of FKBP4 and TMEM173 in BC and normal breast cells. $f$ Representative western blot analysis results of FKBP4, TMEM173 and endogenous control GAPDH. Western blot analysis showed decreased FKBP4 expression and increased TMEM173 expression in T47D and BT549 cells treated by $100 \mathrm{nM}$ naringenin for $24 \mathrm{~h}$. g Representative western blot analysis results of FKBP4, TMEM173 and endogenous control GAPDH. Western blot analysis showed silencing FKBP4 resulted in upregulation of TMEM173 in T47D and BT549 cells. h Representative western blot analysis results of FKBP4-HA, TMEM173 and endogenous control GAPDH. Western blot analysis showed overexpressing FKBP4 resulted in downregulation of TMEM173 in T47D and BT549 cells. i RT-qPCR showed silencing FKBP4 resulted in upregulation of TMEM173 in T47D and BT549 cells ( $n=3$ independent biological replicates). j RT-qPCR showed overexpressing FKBP4 resulted in downregulation of TMEM173 in T47D and BT549 cells ( $n=3$ independent biological replicates). $k$ Luciferase reporter assay showed silencing FKBP4 resulted in increased TMEM173 promoter activity in T47D and BT549 cells ( $n=3$ independent biological replicates). I Luciferase reporter assay showed overexpressing FKBP4 resulted in decreased TMEM173 promoter activity in T47D and BT549 cells ( $n=3$ independent biological replicates). ${ }^{*} p<0.05,{ }^{\star \star} p<0.01,{ }^{* \star *} p<0.001$. 


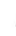

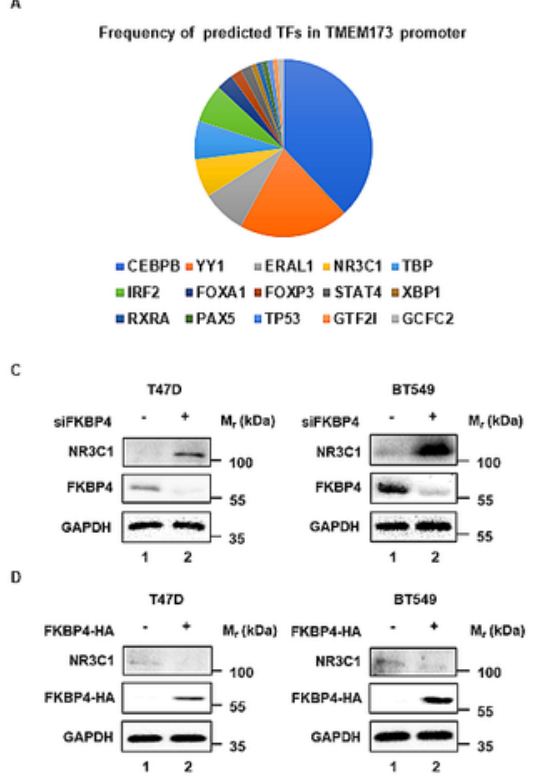

G
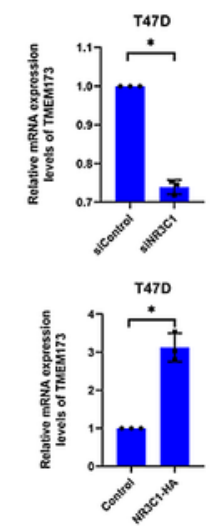

K

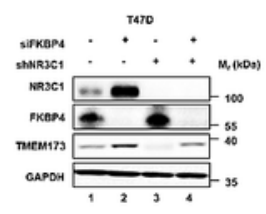

m

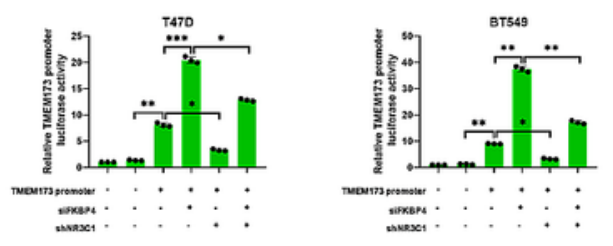

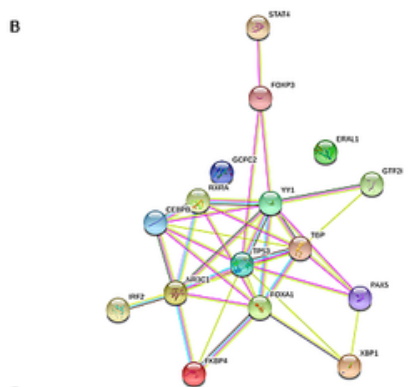
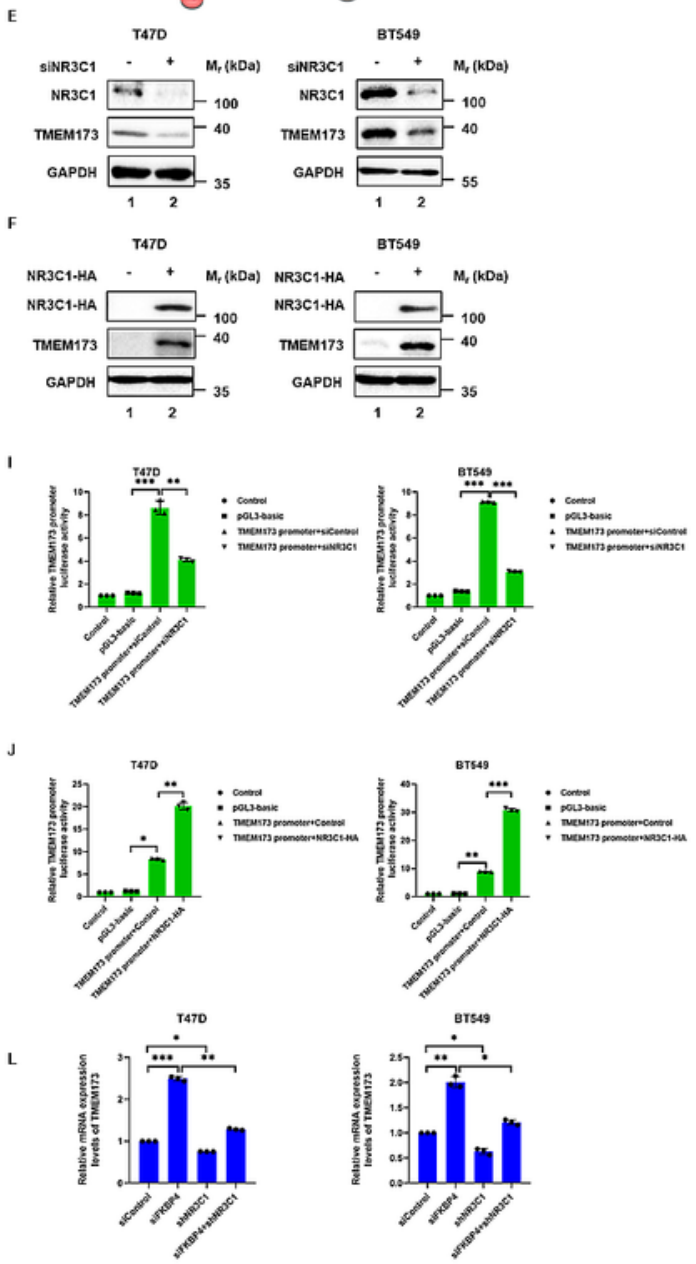

Figure 2

FKBP4 regulates TMEM173 via NR3C1. a Pie chart showed frequency of 15 predicted transcription factors in TMEM173 promoter. $b$ Protein to protein interacting network of FKBP4 and 15 putative TMEM173 transcription factors. c, d Representative western blot analysis results of FKBP4, NR3C1 and endogenous control GAPDH. Western blot analysis showed silencing FKBP4 resulted in upregulation of NR3C1, overexpressing FKBP4 resulted in downregulation of NR3C1 in T47D and BT549 cells. e, f Representative 
western blot analysis results of NR3C1, TMEM173 and endogenous control GAPDH. Western blot analysis showed silencing NR3C1 resulted in downregulation of TMEM173, overexpressing NR3C1 resulted in upregulation of TMEM173 in T47D and BT549 cells. g, h RT-qPCR showed silencing NR3C1 resulted in downregulation of TMEM173, overexpressing NR3C1 resulted in upregulation of TMEM173 in T47D and BT549 cells ( $n=3$ independent biological replicates). i, j Luciferase reporter assay showed silencing NR3C1 resulted in decreased TMEM173 promoter activity, overexpressing NR3C1 resulted in increased TMEM173 promoter activity in T47D and BT549 cells ( $n=3$ independent biological replicates). $k$ Representative western blot analysis results of NR3C1, FKBP4, TMEM173 and endogenous control GAPDH. Western blot analysis showed knockdown of NR3C1 by shRNA attenuated siFKBP4-upregulated TMEM173 expression in T47D and BT549 cells. I RT-qPCR showed knockdown of NR3C1 by shRNA attenuated siFKBP4upregulated TMEM173 expression in T47D and BT549 cells ( $n=3$ independent biological replicates). $m$ Luciferase reporter assay showed knockdown of NR3C1 by shRNA attenuated siFKBP4-upregulated TMEM173 promoter activity in T47D and BT549 cells ( $n=3$ independent biological replicates). ${ }^{\star} p<0.05$, ${ }^{\star \star} p<0.01, * \star \star p<0.001$. 

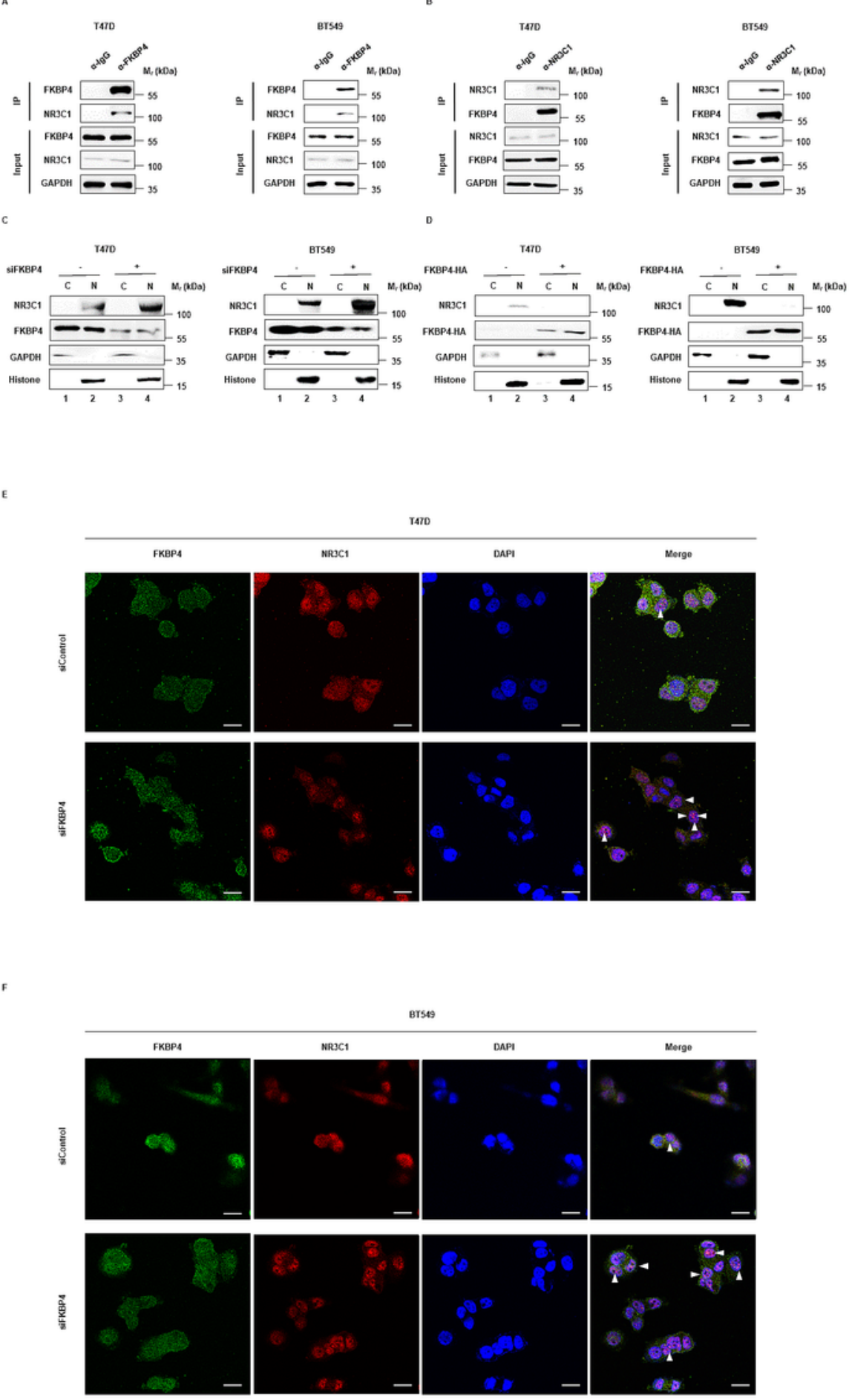

\section{Figure 3}

FKBP4 binds to NR3C1 and FKBP4 inhibition triggers nuclear translocation of NR3C1. a, b co-IP and western blot assays using antibodies as indicated for binding between endogenous FKBP4 and NR3C1 in T47D and BT549 cells. c, d T47D and BT549 cells were transfected with siRNAs or plasmids. Cells were harvested, and the nuclear and cytoplasmic fractions were separated. Proteins were analyzed by immunoblotting using anti-NR3C1, anti-FKBP4, anti- GAPDH, or anti-histone antibody ( $\mathrm{n}=3$ independent 
biological replicates). e, f T47D and BT549 cells were transfected with siRNAs. Immunofluorescent staining was carried out using anti-NR3C1 and anti-FKBP4. DAPI staining was performed to show the nuclei ( $n=3$ independent biological replicates). The arrowheads show the NR3C1 nuclear localization under FKBP4 inhibition. Scale bar=20 $\mu$ m.
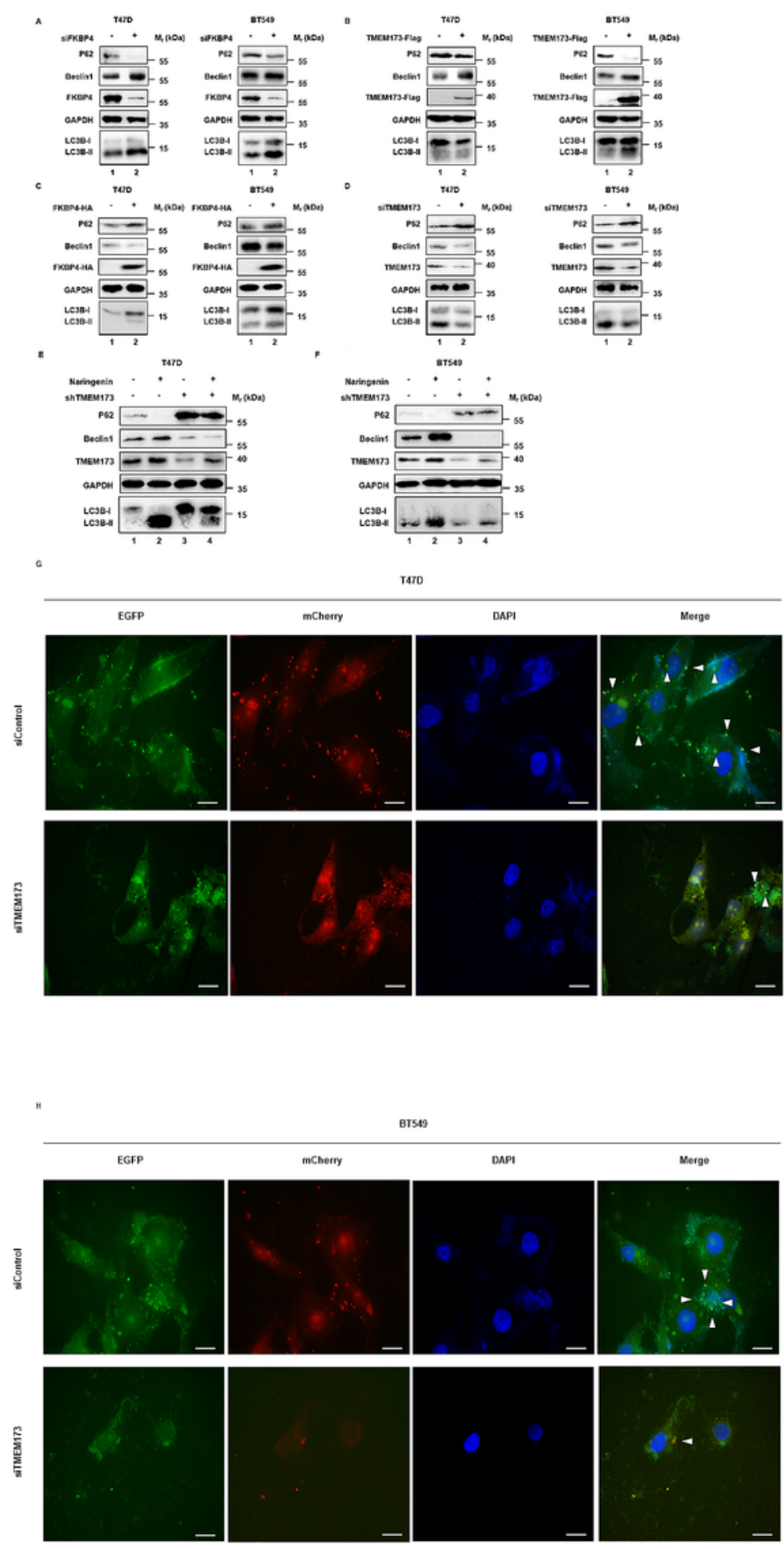

Figure 4 
FKBP4/NR3C1/TMEM173 axis is involved in naringenin-induced autophagy. a Representative western blot analysis results of P62, Beclin1, FKBP4, LC3B and endogenous control GAPDH. Western blot analysis showed silencing FKBP4 resulted in downregulation of P62 and upregulation of Beclin1 and LC3B-II/LC3BI in T47D and BT549 cells. b Representative western blot analysis results of P62, Beclin1, TMEM173-Flag, LC3B and endogenous control GAPDH. Western blot analysis showed overexpressing TMEM173 resulted in downregulation of P62 and upregulation of Beclin1 and LC3B-II/LC3B-I in T47D and BT549 cells. C Representative western blot analysis results of P62, Beclin1, FKBP4-HA, LC3B and endogenous control GAPDH. Western blot analysis showed overexpressing FKBP4 resulted in upregulation of P62 and downregulation of Beclin1 and LC3B-II/LC3B-I in T47D and BT549 cells. d Representative western blot analysis results of P62, Beclin1, TMEM173, LC3B and endogenous control GAPDH. Western blot analysis showed silencing TMEM173 resulted in upregulation of P62 and downregulation of Beclin 1 and LC3BII/LC3B-I in T47D and BT549 cells. e, f Representative western blot analysis results of P62, Beclin1, TMEM173, LC3B and endogenous control GAPDH. Western blot analysis showed knockdown of TMEM173 by shRNA attenuated naringenin-induced autophagy in T47D and BT549 cells treated by 100 $\mathrm{nM}$ naringenin for $24 \mathrm{~h} . \mathrm{g}$, h Measurement of autophagy flux in T47D and BT549 cells co-transfected with p-mCherry-C1-EGFP-hLC3B and siTMEM173. Scale bar=20 $\mu \mathrm{m}$. 

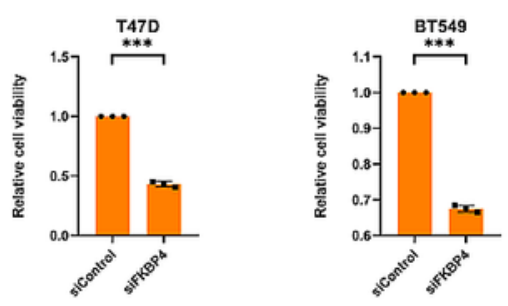

c
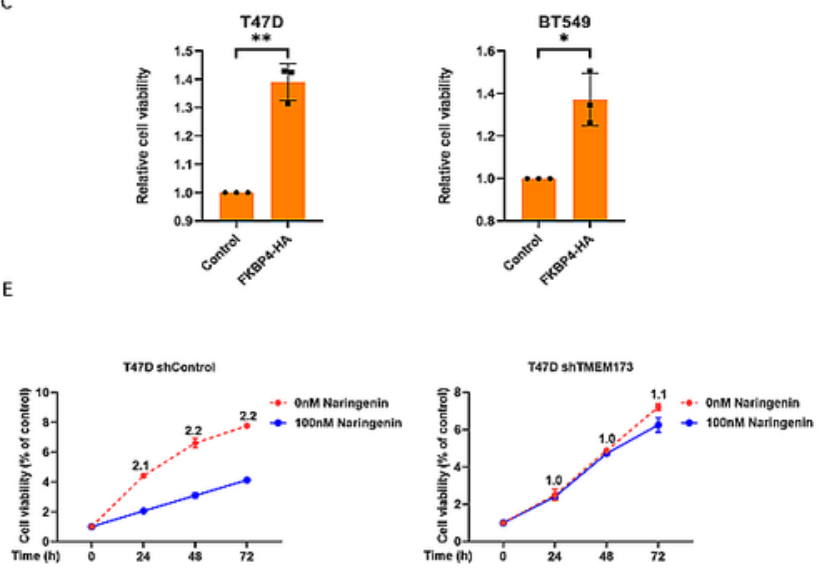

G
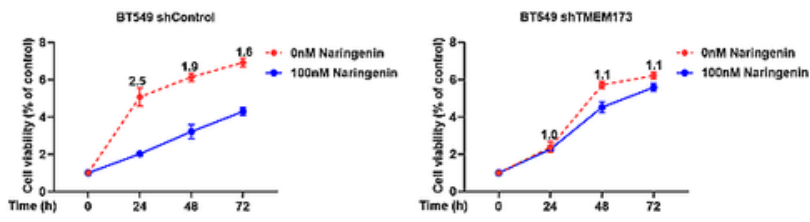

D
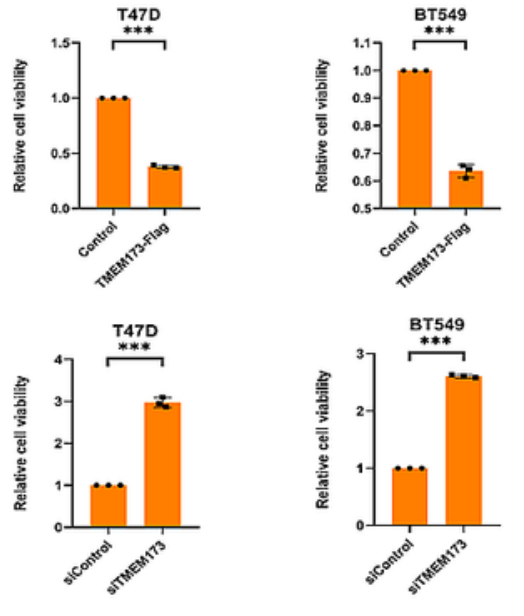

F

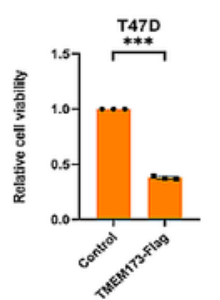

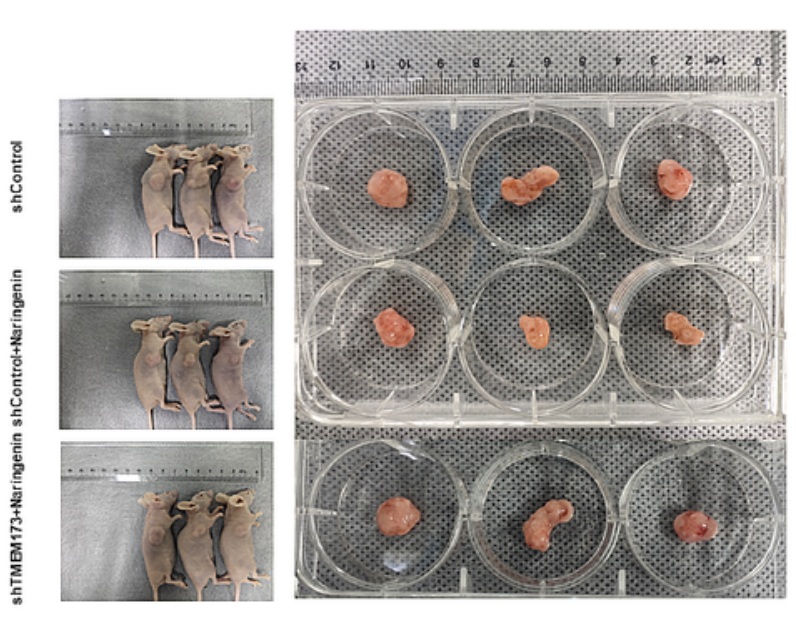
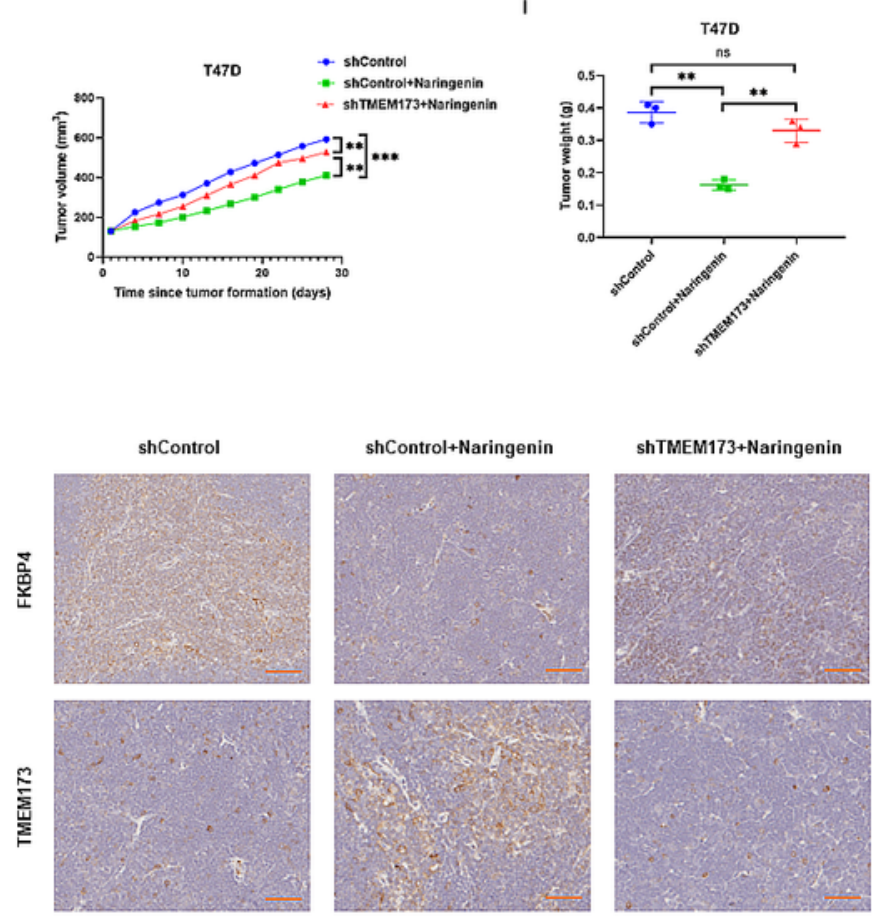

\section{Figure 5}

FKBP4/NR3C1/TMEM173 axis is involved in naringenin-restrained cell proliferation. a Bar chart showed silencing FKBP4 resulted in decreased cell viability of T47D and BT549 cells at $72 \mathrm{~h}$ ( $\mathrm{n}=3$ independent biological replicates). b Bar chart showed overexpressing TMEM173 resulted in decreased cell viability of T47D and BT549 cells at $72 \mathrm{~h}$ ( $\mathrm{n}=3$ independent biological replicates). $\mathrm{c} \mathrm{Bar}$ chart showed overexpressing FKBP4 resulted in increased cell viability of T47D and BT549 cells at $72 \mathrm{~h}(\mathrm{n}=3$ independent biological 
replicates). d Bar chart showed silencing TMEM173 resulted in increased cell viability of T47D and BT549 cells at $72 \mathrm{~h}$ ( $\mathrm{n}=3$ independent biological replicates). e, $\mathrm{f}$ Line chart showed knockdown of TMEM173 by shRNA attenuated naringenin-restrained cell proliferation in T47D and BT549 cells treated by $100 \mathrm{nM}$ naringenin, numeric representation at each dot is cell viability fold change of $0 \mathrm{nM}$ and $100 \mathrm{nM}$ naringenin treatment ( $n=3$ independent biological replicates). $g, h$, $i$ The macroscopic appearance, volume and weight of subcutaneous tumors in mice ( $n=3$ /group) transplanted with T47D cells treated with shControl, shControl+Naringenin and shTMEM173+Naringenin. $j$ The expression of FKBP4 and TMEM173 in mice transplanted with T47D cells treated with shControl, shControl+Naringenin and shTMEM173+Naringenin were determined by using immunohistochemistry. ${ }^{*} p<0.05,{ }^{*} p<0.01,{ }^{* * *} p<0.001$. 
A

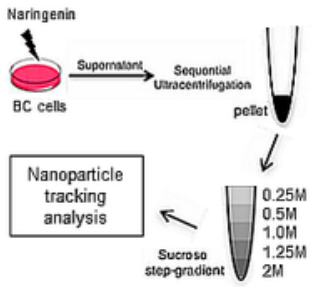

B

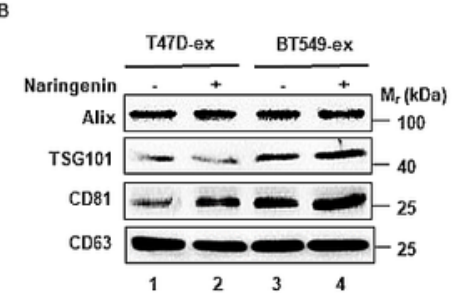

D

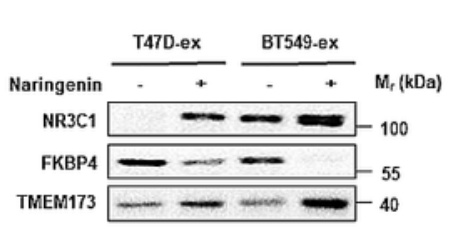

E

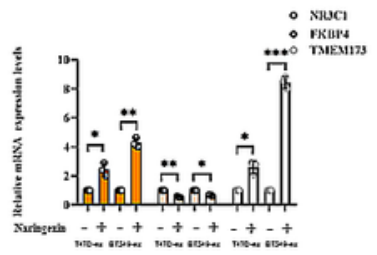

G

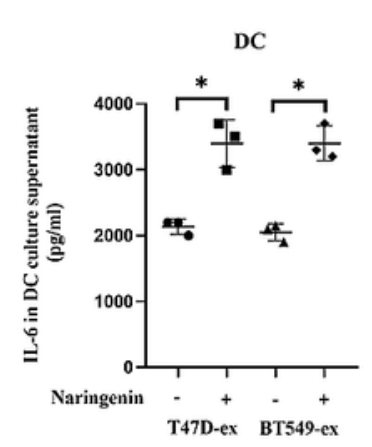

DC

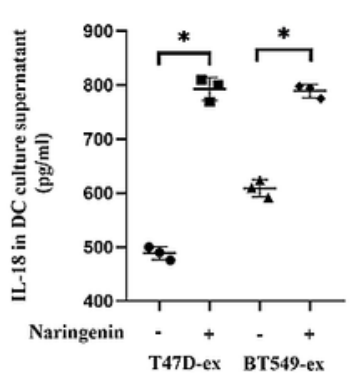

H
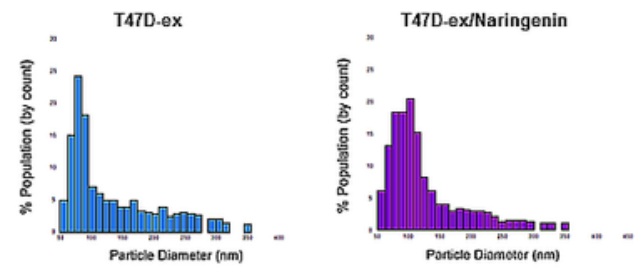

BT549-ex
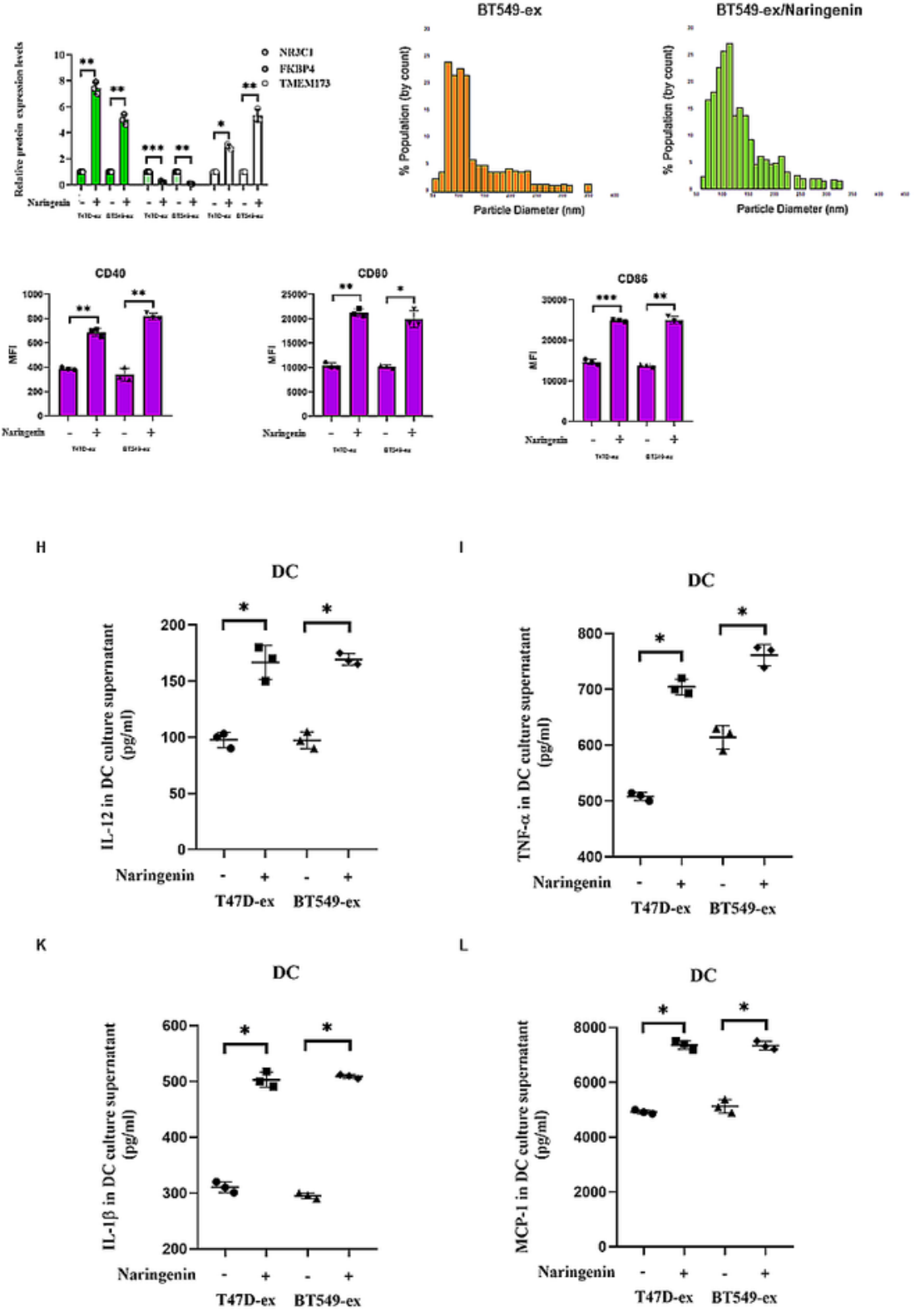

\section{Figure 6}

Identified breast cancer exosomes promote dendritic cell maturation. a Schema of breast cancer exosomes isolation method. b Western blot analysis of exosome biomarkers (Alix, TSG101, CD81 and CD63). c Particle size distribution of exosomes measured by Nanoparticle tracking analysis. $d, e$ The expression of NR3C1, FKBP4 and TMEM173 in T47D-ex and BT549-ex were examined by using western blot analysis and RT-qPCR. $f$ CD11 c+ DCs were cultured for $72 \mathrm{~h}$ with T47D-ex and BT549-ex $(30 \mu \mathrm{g})$ and 
analyzed for expression of co-stimulatory molecules by flow cytometry analysis. Mean fluorescence intensity (MFI) \pm s.e.m. of samples in each group ( $\mathrm{n}=3$ independent biological replicates). MFI was calculated after subtracting background. g-I The level of cytokines IL-6, IL-12, TNF-a, IL-18, IL-1 $\beta$ and MCP1 secretion in DC culture supernatants were measured by ELISA. ${ }^{*} p<0.05,{ }^{*} p<0.01,{ }^{* *} p<0.001$.
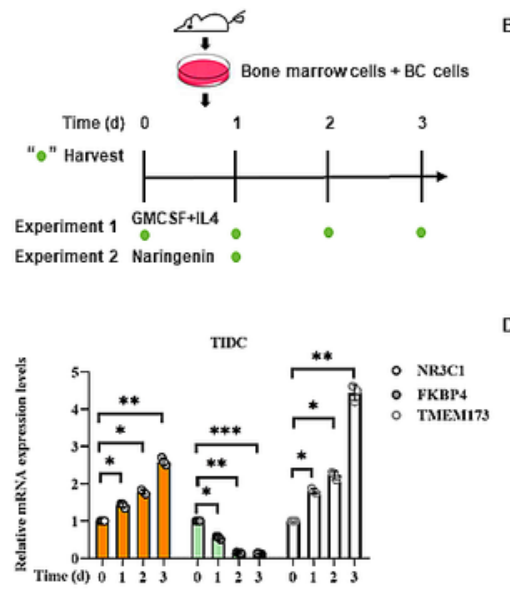

B
$\mathrm{E}$
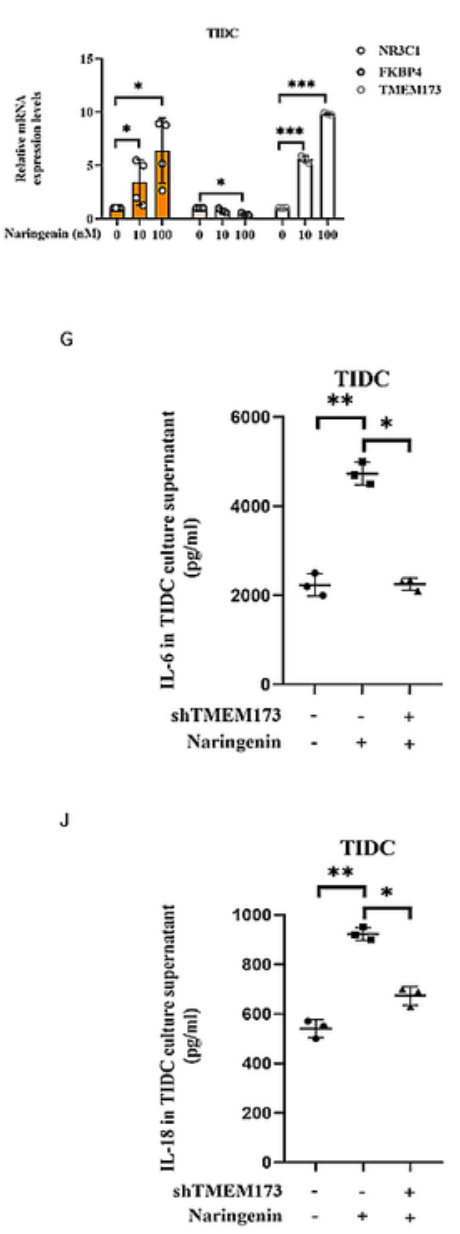

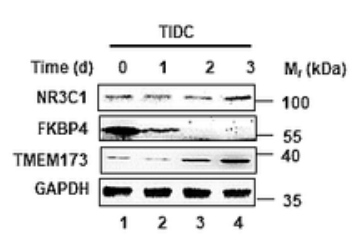

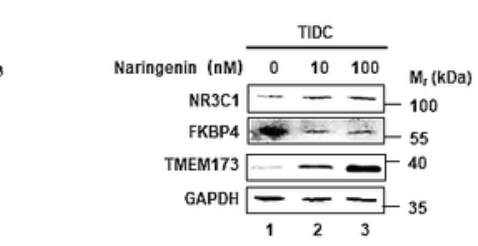
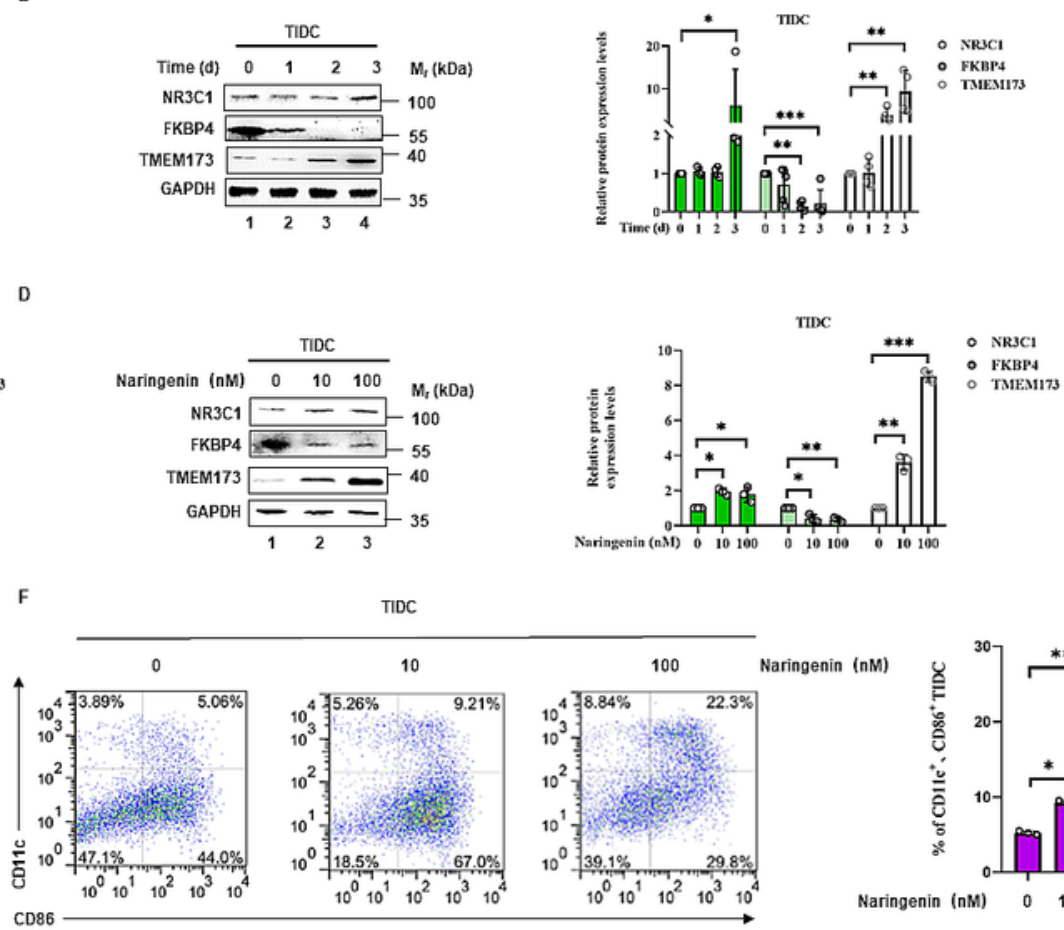

Naringenin (nM)

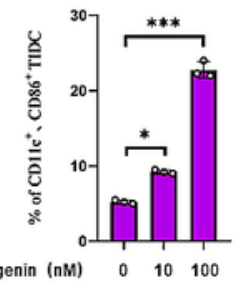

H

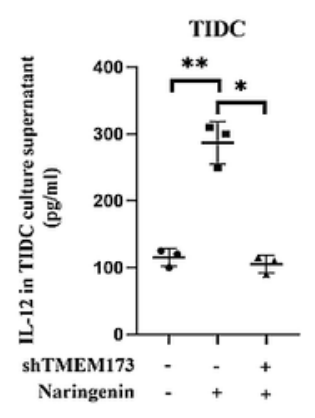

K

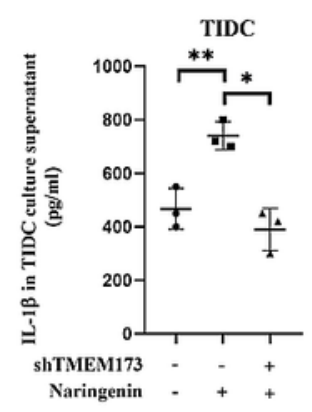

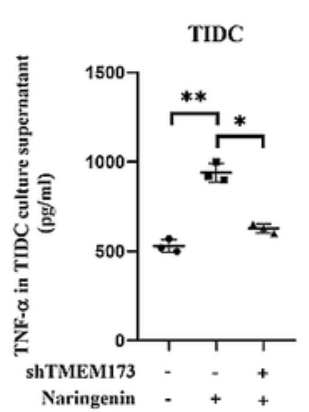

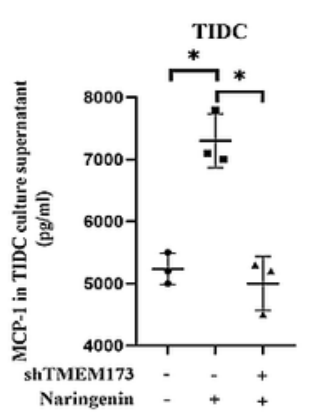

Figure 7 
FKBP4/NR3C1/TMEM173 signaling pathway involved in naringenin-induced TIDC maturation. a Schema of mice bone marrow cells co-cultured with BC cells. b Western blot analysis of NR3C1, FKBP4 and TMEM173 protein expression in DC co-cultured with BC cells for 3 days. C RT-qPCR analysis of NR3C1, FKBP4 and TMEM173 mRNA expression in DC co-cultured with BC cells for 3 days. $d$, e The expression of NR3C1, FKBP4 and TMEM173 in TIDC on different concentration of naringenin were examined by using western blot analysis and RT-qPCR. $f$ TIDC were treated with different concentration of naringenin for $72 \mathrm{~h}$ and analyzed for maturation by flow cytometry analysis. g-I The level of cytokines IL-6, IL-12, TNF-a, IL-18, IL-1 $\beta$ and MCP-1 secretion in TIDC culture supernatants were measured by ELISA. ${ }^{*} p<0.05,{ }^{* *} \mathrm{p}<0.01$, $* \star * p<0.001$.

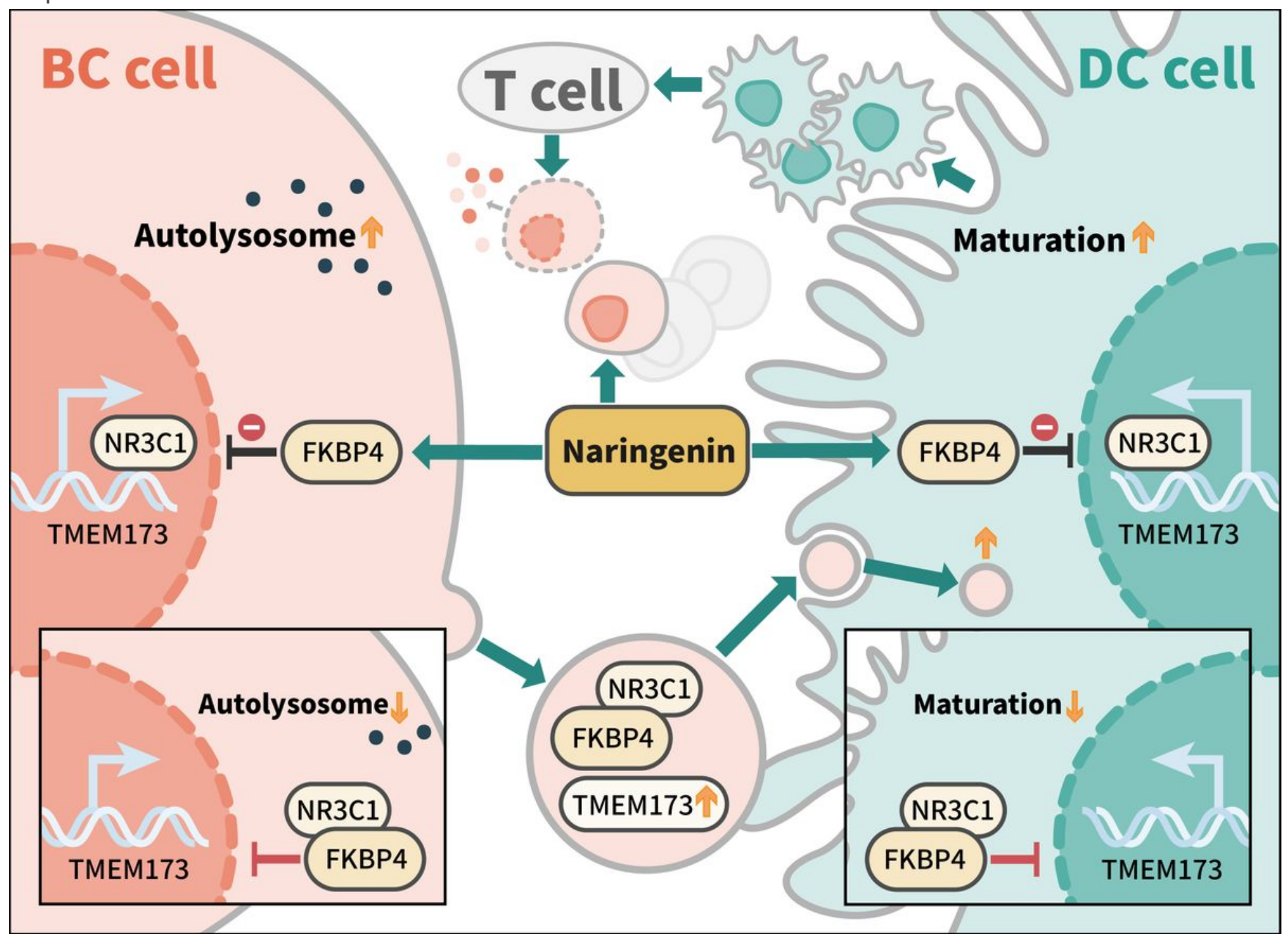

Figure 8

Model of naringenin-mediated cell proliferation inhibition and cytoprotective autophagy of $\mathrm{BC}$ cells and TIDC maturation promotion through FKBP4/NR3C1/TMEM173 signaling pathway. Naringenin inhibits FKBP4, thus releasing and inducing NR3C1 to bind to the TMEM173 gene promoter, which triggers upregulation of TMEM173 mRNA and protein level, leading to proliferation inhibition and autophagy promotion of BC cells, as well as maturation promotion of TIDC. Among tumor microenvironment, 
naringenin could regulate the expression of FKBP4, NR3C1 and TMEM173 carried by BC exosomes, thus enhancing maturation and antigen presenting function of TIDC.

\section{Supplementary Files}

This is a list of supplementary files associated with this preprint. Click to download.

- S1.png

- S2.png

- S3.png

- S4.png

- S5.png

- S6.png

- S7.png

- S8.png

- S9.png

- S10.png 